\title{
Complex collisions of ultracold molecules: A toy model
}

\author{
Jia K. Yao $\odot,{ }^{1,{ }^{*}}$ Cooper A. Johnson $\odot,{ }^{2, \dagger}$ Nirav P. Mehta $\odot,{ }^{2, \ddagger}$ and Kaden R. A. Hazzard $\odot^{3,4, \S}$ \\ ${ }^{1}$ Department of Physics, California Institute of Technology, Pasadena, California 91125, USA \\ ${ }^{2}$ Trinity University, One Trinity Place, San Antonio, Texas 78212-7200, USA \\ ${ }^{3}$ Department of Physics and Astronomy, Rice University, Houston, Texas 77005, USA \\ ${ }^{4}$ Rice Center for Quantum Materials, Rice University, Houston, Texas 77005, USA
}

(Received 2 August 2021; accepted 15 October 2021; published 10 November 2021)

\begin{abstract}
We introduce a model to study the collisions of two ultracold diatomic molecules in one dimension interacting via pairwise potentials. We present results for this system and argue that it offers lessons for real molecular collisions in three dimensions. We analyze the distribution of the adiabatic potentials in the hyperspherical coordinate representation as well as the distribution of near-threshold four-body bound states, systematically studying the effects of molecular properties, such as interaction strength, interaction range, and atomic mass. It is found that the adiabatic potential's nearest-neighbor energy level distribution transitions from significant level repulsion characteristic of chaos (Brody distribution) to nonchaotic (Poisson distribution) as the two molecules are separated. For the near-threshold four-atom bound states, the case where all atoms have equal masses shows a Poissonian spacing distribution, while the unequal-mass system exhibits significant level repulsion characterized by a nonzero Brody parameter. We derive a semiclassical formula for the density of states and extract from it simple scaling laws with potential depth and range. We find good agreement between the semiclassical predictions for the density of states and the full quantum mechanical calculations.
\end{abstract}

DOI: 10.1103/PhysRevA.104.053311

\section{INTRODUCTION}

Ultracold molecules have emerged as a new platform for quantum science and technology [1,2]. They combine the unique, tunable, and coherent setting of ultracold matter with strong dipolar interactions and numerous stable rotational and vibrational states. Consequently, this platform has wide-ranging applications, including exploring new phases of matter and nonequilibrium behavior [3-25,25-35] (for reviews, see Refs. [1,2,36-39]), enabling quantum computation [40-45], performing precision measurements, such as measuring the electron electric dipole moment [46-50], and studying chemical reactions in the quantum regime [51-60].

To fully realize these applications, it is necessary to theoretically understand the collisional behavior of molecules. For example, the density of bimolecular bound states at the collision energy is important in determining the lifetime of a cloud of molecules $[51,53,61,62]$, the many-body physics in an optical lattice [63-66], and chemical reaction rates. In addition to being necessary for applications, understanding molecular collisions is of fundamental scientific interest. Molecules are an intermediate step between atoms, which are mostly simple and nonchaotic, and thermodynamically large systems, which frequently exhibit chaotic behavior. The important question then arises of whether and how quantum chaos occurs in

*jyyao@caltech.edu

†johns10@trinity.edu

‡nmehta@trinity.edu

§kaden.hazzard@gmail.com molecular collisions. It is believed that quantum systems which become chaotic in the classical limit display spectral fluctuations identical to those of random matrices generated from certain classes of statistical ensembles in random matrix theory [67-69]. A chaotic time-reversal symmetric system is expected to be described by a Gaussian orthogonal ensemble (GOE), with a characteristic level repulsion described by the Wigner-Dyson distribution. On the other hand, levels of nonchaotic, integrable systems follow a Poisson distribution. Manifestations of random matrix theory are well studied in nuclear spectra and collisions [70-72].

Molecular collisions are significantly more complex than atomic collisions because molecules have a much denser collection of electronic, rotational, vibrational, and hyperfine states, resulting in a density of resonances that is several orders of magnitude higher. This was suggested by Mayle et al. [73,74], and recently estimated by Christianen et al. [75] to be $0.124 \mu \mathrm{K}^{-1}$ for $\mathrm{NaK}+\mathrm{NaK}$ collision complexes, without including the hyperfine states that will further increase the resonance density. Thus, there will exist many accessible closed-channel bound states around the collision energyeven at the coldest available experimental temperatures. Consequently, while scattering resonances are routinely measured in ultracold atoms [76], even for atoms with the densest resonance spectra, such as those explored in lanthanide atoms (Dy and Er) [77-86] and predicted in alkaline-earth-like atoms ( $\mathrm{Yb})$ [87], it is much harder to resolve the resonances in molecular collisions due to the significantly higher density of states. In addition to being difficult to experimentally measure, molecule-molecule collisions are extremely challenging to model, and quantitative calculations remain impossible 
for most diatomic molecules, including bialkali molecules. Recent work has emphasized the high resonance density inherent to bialkali (and heavier) atom-molecule [63-65,88-93] and molecule-molecule [94-96] collisions, building on earlier research on molecules formed from lighter atoms [61,97105].

More recently, Christianen et al. [106] theoretically demonstrated that in addition to sticky collisions, photoexcitation by the trapping laser can significantly contribute to the collisional loss of ground-state bialkali dimers. Since then, a series of recent experiments confirm such photoinduced loss in both reactive and nonreactive molecule-molecule and atom-molecule collisions [107-109]. Experiments studying different bialkali species [110-112] suggest that the complex lifetime may be underestimated or that there are unknown loss mechanisms beyond photoinduced loss. The control of dipolar interactions via microwave dressing [113,114] and electric fields [115-118] is being studied, including the interaction effects on molecule loss.

Despite the collisional loss from photoexcitations, open questions remain in current studies of sticky collisions, such as the following: Is chaos universal in molecule collisions? What is the effect of the molecular size, the mass of the constituent atoms, and the type of interactions?

In this paper, we present a highly simplified model of the collision of two identical molecules, which can shed light on the questions above, while at the same time being amenable to straightforward numerical calculation. The principal simplification is to restrict the atoms to move in one dimension, interacting via a simple model potential (either Morse or Pöschl-Teller). This potential is chosen to be deep enough to harbor many two-atom bound states, mimicking the situation in real molecules. For concreteness, we choose each molecule to be composed of two distinguishable fermionic isotopes.

We find several results that may have implications for scattering of real molecules in three dimensions. These results can be divided into three categories: (1) results associated with the statistics of the adiabatic potential curves, (2) results associated with the statistics of four-body bound states, and, finally, (3) results stemming from a semiclassical analysis of the four-atom density of states.

First, we find that the statistics of the adiabatic potentials transition from chaotic to nonchaotic as the intermolecular separation increases, similar to results observed for atommolecule scattering in calculations using realistic potential energy surfaces [92]. We characterize how the detailed behavior of this crossover depends on the parameters of the model potential. We also show that there are clear trends in the average spacing of the adiabatic potentials and the chaoticity of their level-spacing statistics as a function of hyper-radius, collision energy, molecular mass, and length scale of the potential. We expect that these qualitative trends persist to three-dimensional systems.

The second, more surprising, finding results from calculating the four-body bound states. We find that for the equal-mass case, four-body level statistics show no apparent chaos even when the adiabatic potentials are strongly chaotic. We trace the resulting Poisson statistics to the presence of a symmetry that is broken when the masses are taken to be different. For the unequal-mass case, we observe strong level repulsion in the four-body spectrum, resulting in a nonzero Brody parameter. The case with emergent symmetry suggests caution when interpreting the level statistics of adiabatic potentials, which may not correspond to the statistics in the spectrum of the four-body bound states. Lastly, and perhaps most interestingly, we derive a analytic semiclassical relation between the four-body density of states and the two-atom interaction parameters. We show that although the derivation relies on approximations, they accurately capture the scaling with interaction parameters and mass ratio. This suggests that the analogous expressions for the three-dimensional system will allow one to simply estimate the effects of changing molecular species or other experimental parameters on complex molecule-molecule collisions.

The structure of this paper is as follows. Section II describes the problem we solve and the tools associated with the hyperspherical coordinates we use to numerically obtain the adiabatic potentials. Section III presents the results for the adiabatic potentials. Section IV shows the density of fourbody bound states and their nearest-neighbor level statistics obtained by numerically solving the four-particle Schrödinger equation using a slowly variable discretization [119] (SVD) approach with the radial coordinate treated using a discrete variable representation (DVR). It derives approximate analytic expressions for the scaling of the four-body density of states and shows that despite the approximations involved, they agree well with the numerical results. Section V summarizes the results and gives an outlook, including the relevance of these results to real three-dimensional molecule-molecule scattering.

\section{MOLECULE-MOLECULE SCATTERING AND HYPERSPHERICAL COORDINATES}

We study a system of two identical diatomic molecules in one dimension. Each molecule consists of two fermionic atoms, giving two sets of identical fermions in total, labeled as $(1,2)$ and $(3,4)$. We take 1 and 3 to be distinguishable from 2 and 4. The masses of the distinguishable atoms are either set to be equal or different by a small amount. Throughout the paper, we fix the mass of atom 1 (and 2), i.e., $m_{1}=m_{2}=1$, and vary the mass of atom 3 (and 4 ) by setting $m_{3} / m_{1}=1$, 1.3 , or 1.5 .

We choose a system of dimensionless variables in all that follows. Distances such as atom separation $r$ and hyper-radius $R$ are scaled by an arbitrary length scale $L$. Energy scales such as the two-body potential depth $D$ are scaled by $E_{L}=$ $\hbar^{2} /\left(m_{1} L^{2}\right)$.

We present results for pairwise atom-atom interactions with two potentials, i.e., Morse and Pöschl-Teller potentials,

$$
\begin{aligned}
U_{\text {Morse }}(r) & =D\left[1-e^{-a\left(r-r_{0}\right)}\right]^{2}-D, \\
U_{\text {Pöschl-Teller }}(r) & =-\frac{D}{\cosh ^{2}\left(r / r_{0}\right)},
\end{aligned}
$$

where $r \geqslant 0$ is the interatomic distance, $D$ is the depth of the potential, $r_{0}$ is the width of the potential, and $a$ is set to $1 / r_{0}$, so that $U(0) / D$ is independent of $r_{0}$. In either case, the model potential is assumed to be identical for all pairs of atoms. 
Before continuing, we comment on the drastic simplifications being made, the first of which is our choice of model potentials. Although these potentials are highly simplified compared to realistic interatomic interactions, they capture characteristic short-range repulsion and a finite bond distance, which are crucial qualitative features. Of course, many potentials satisfy these simple requirements and these two are chosen somewhat arbitrarily and for simplicity.

The primary limitation of these potentials is that they fail to capture the long-range van der Waals $C_{6} / r^{6}$ behavior. Adding such a potential can qualitatively affect the near-threshold behavior of bound states and scattering (even beyond that described by effective range theory; see, e.g., Ref. [120]), but it has little effect on bound states that are bound more deeply than the van der Waals energy. While such near-threshold physics is an important component of ultracold scattering, a simpler treatment ignoring the $C_{6} / r^{6}$ tail still offers a valuable perspective for two reasons. Most importantly, there is a rich and experimentally relevant bound state spectrum away from the near-threshold energy window.

The second, and by far the most drastic, simplification is the dimensionality one dimension (1D) versus 3D for real molecules. Its most important qualitative differences are the expected greater density of states in 3D and the existence of more symmetry sectors (e.g., angular momentum, as well as internal states such as hyperfine states). A smaller difference is the modification of the Wigner threshold laws [121], but these are expected to play a relatively small role in the physics we focus on, as argued above. While these limitations make any direct comparison with real molecules impossible, the payoff of the simplifications is rendering the problem tractable, while allowing qualitative features-scaling of the four-body bound state density of states, and level statistics of the adiabatic channels and four-body bound states-to be studied, providing indirect insights into possible behaviors to explore in real molecules.

We use hyperspherical coordinates to solve the Schrödinger equation and interpret its eigenstates. Hyperspherical coordinates have been used widely [89,122132] to study few-body physics and provide an efficient and powerful method for studying the full scattering and bound state problem. In this representation, the particle coordinates are rewritten into one hyper-radial coordinate and a collection of angular coordinates (defined below). In addition to these calculations, in Sec. IV B we employ a SVD [119] approach, treating the radial coordinate using a DVR [133], which allows us to obtain the full four-body eigenstates.

In one dimension, the position and the mass of particle $i$ in the laboratory frame are labeled by $r_{i}$ and $m_{i}$, respectively. By setting the center-of-mass coordinate to zero, the remaining three degrees of freedom can be expressed in terms of Jacobi coordinates $y_{1}, y_{2}$, and $y_{3}$, defined as

$$
\begin{aligned}
& y_{1}=\sqrt{\frac{\mu_{12}}{\mu}}\left(r_{1}-r_{2}\right), \\
& y_{2}=\sqrt{\frac{\mu_{34}}{\mu}}\left(r_{3}-r_{4}\right), \\
& y_{3}=\sqrt{\frac{\mu_{12,34}}{\mu}}\left(\frac{m_{1} r_{1}+m_{2} r_{2}}{m_{1}+m_{2}}-\frac{m_{3} r_{3}+m_{4} r_{4}}{m_{3}+m_{4}}\right),
\end{aligned}
$$

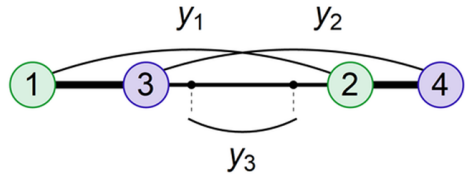

FIG. 1. Jacobi coordinates $y_{1}, y_{2}$, and $y_{3}$ for the four-fermion system in one dimension, where the center-of-mass coordinate is set to 0 . Particles 1 and 2 are indistinguishable, and particles 3 and 4 are indistinguishable. When $(1,3)$ and $(2,4)$ are coincident, and the separation between these molecules is large, the hyperspherical coordinates approach $(\phi, \theta) \rightarrow(\pi / 4, \pi / 2)$ for $m_{1}=m_{2}=m_{3}=m_{4}$.

where

$$
\begin{aligned}
\mu_{12} & =\frac{m_{1} m_{2}}{m_{1}+m_{2}}, \\
\mu_{34} & =\frac{m_{3} m_{4}}{m_{3}+m_{4}}, \\
\mu_{12,34} & =\frac{\left(m_{1}+m_{2}\right)\left(m_{3}+m_{4}\right)}{m_{1}+m_{2}+m_{3}+m_{4}}, \\
\mu & =\left(m_{1} m_{2} m_{3} m_{4}\right)^{1 / 4} .
\end{aligned}
$$

Figure 1 illustrates these Jacobi coordinates for the system studied in this paper. The hyperspherical coordinates $(R, \phi, \theta)$ are related to the Jacobi coordinates by

$$
\begin{aligned}
& y_{1}=R \cos \phi \sin \theta, \\
& y_{2}=R \sin \phi \sin \theta, \\
& y_{3}=R \cos \theta,
\end{aligned}
$$

where the hyper-radius is defined as

$$
R=\sqrt{y_{1}^{2}+y_{2}^{2}+y_{3}^{2}} .
$$

In this formalism, the hyper-radius $R$ effectively characterizes the size of the system, and the angular coordinates $\theta$ and $\phi$ describe the relative distances between pairs of atoms. For example, as $(\phi, \theta) \rightarrow(\pi / 4, \pi / 2)$, the system is separated into two constituent parts, $(1,3)$ and $(2,4)$. When the system is in a state of two well-separated molecules, $R$ measures the intermolecular separation. In this coordinate system, the exchange and parity symmetries of the system can also be readily imposed via boundary conditions for $\theta$ and $\phi$, as discussed in Appendix A. For further information on the hyperspherical representation, refer to Ref. [132].

With the hyperspherical coordinates, the Schrödinger equation of the four-body system in 1D can be decomposed into a hyperangular component and a hyper-radial component, giving the hyperspherical Schrödinger equation for the reduced wave function $\psi(R, \theta, \phi)=R \Psi(R, \theta, \phi)$ [where $\Psi(R, \theta, \phi)$ is the wave function, and we set $\hbar=1$ throughout this paper],

$$
\left[-\frac{1}{2 \mu} \frac{\partial^{2}}{\partial R^{2}}+\hat{H}_{\mathrm{ad}}(R, \theta, \phi)\right] \psi(R, \theta, \phi)=E \psi(R, \theta, \phi),
$$

where $\hat{H}_{\text {ad }}$ is the adiabatic Hamiltonian,

$$
\hat{H}_{\mathrm{ad}}(R, \theta, \phi)=\frac{\hat{L}^{2}(\theta, \phi)}{2 \mu R^{2}}+\hat{V}(R, \theta, \phi),
$$

$\hat{L}$ is the (hyper)angular momentum operator, and $\hat{V}$ is the interaction between the particles. $\hat{V}$ is obtained by summing 
up all the pairwise interactions $U(r)$ for each pair of molecules with separation $r$, i.e., $V(R, \theta, \phi)=\sum_{i>j} U\left(r_{i j}\right)$. The hyperspherical adiabatic potentials are defined as the eigenvalues of the adiabatic Hamiltonian $H_{\mathrm{ad}}$,

$$
\hat{H}_{\mathrm{ad}}(R, \theta, \phi) \Phi_{v}(R ; \theta, \phi)=U_{v}(R) \Phi_{v}(R ; \theta, \phi) .
$$

Such hyperspherical adiabatic curves play a large role in understanding few-body scattering. Section III offers an analysis of these adiabatic potentials.

To solve the Schrödinger equation for the four-body eigenstates, we build an exact reformulation in terms of the adiabatic potentials $U_{v}(R)$ using a slow variable discretization [119] (SVD). The essence of the method is to treat the radial coordinate first using a discrete variable representation (DVR) [133], then solve the rest of the problem in terms of these.

We expand the full wave function as a sum over DVR basis functions with $\Omega$-dependent coefficients,

$$
\Psi(R, \Omega)=\sum_{i} \chi_{i}(R) \tilde{\Phi}_{i}(\Omega) .
$$

Here, the radial basis functions $\chi_{i}(R)$ are constructed from the Gauss-Lobatto shape functions. They are defined in terms of rescaled Gauss-Lobatto integration weights $w_{i}$ and nodes $R_{i}$ [133-136],

$$
\chi_{i}(R)=w_{i}^{-1 / 2} \prod_{j \neq i} \frac{R-R_{j}}{R_{i}-R_{j}} .
$$

When evaluated at any of the $N_{R}$ integration points, $R_{j}$, these radial basis functions have the remarkable DVR property $\chi_{i}\left(R_{j}\right)=\delta_{i j}$. Only the $i=1$ and $i=N_{R}$ basis functions are nonzero at the boundaries, so to impose boundary conditions $\Psi(R=0, \Omega)=\Psi\left(R=R_{\max }, \Omega\right)=0$, one simply omits those functions from the basis set. The resulting radial set has $N_{R}-2$ members. The $\Omega$-dependent coefficient $\tilde{\Phi}_{i}(\Omega)$ in Eq. (9) is expanded in the adiabatic basis $\Phi_{v}(R ; \Omega)$ at each of the Gauss-Lobatto integration points $R_{j}$ as

$$
\tilde{\Phi}_{j}(\Omega)=\sum_{\mu} C_{j \mu} \Phi_{\mu}\left(R_{j} ; \Omega\right),
$$

resulting in a set of coupled equations,

$$
\sum_{j, \mu} T_{i j} O_{i v, j \mu} C_{j \mu}+U_{v}\left(R_{i}\right) C_{i v}=E C_{i v},
$$

where

$$
O_{i v, j \mu}=\left\langle\Phi_{\nu}\left(R_{i}\right) \mid \Phi_{\mu}\left(R_{j}\right)\right\rangle,
$$

and the matrix elements

$$
T_{i j}=\frac{\hbar^{2}}{2 \mu} \int_{0}^{r_{\max }} \frac{d \chi_{i}}{d R} \frac{d \chi_{j}}{d R} d R
$$

are calculated by the $N_{R}$-point Gauss-Lobatto quadrature rule. The derivatives of the basis functions are calculated at each of the integration points according to the formula [135-137]

$$
\left[\frac{d \chi_{i}}{d R}\right]_{R=R_{j}}= \begin{cases}\frac{w_{i}^{-1 / 2}}{R_{i}-R_{j}} \prod_{k \neq j, i}^{N_{R}} \frac{R_{j}-R_{k}}{R_{i}-R_{k}} & (i \neq j) \\ \frac{\delta_{i, N_{R}}-\delta_{i, 1}}{2 \omega_{i}^{3 / 2}} & (i=j) .\end{cases}
$$

Solutions to Eq. (12) yield the four-body bound state spectrum, from which we obtain the four-body density of states and level-spacing distribution. Our numerical calculations
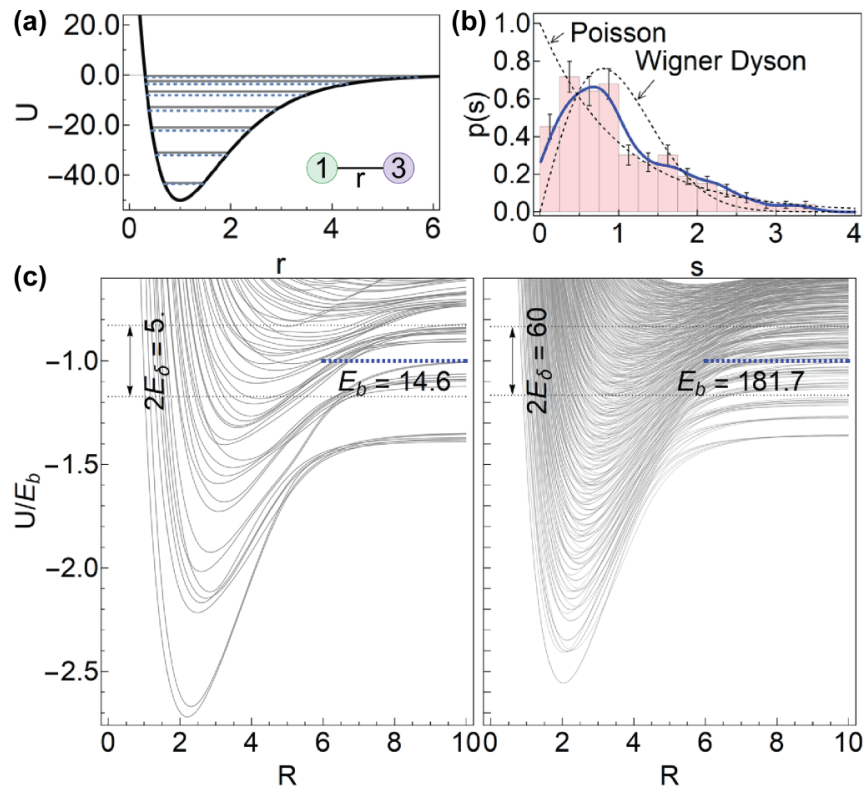

FIG. 2. (a) Bound states of the two-atom interaction between atoms 1 and 3 in a Morse potential, given by Eq. (1a), for $D=$ 50, $a=1 / r_{0}$, and $r_{0}=1$. Energy levels for $m_{3} / m_{1}=1$ (solid) and $m_{3} / m_{1}=1.3$ (dashed) are shown. (b) Probability distribution $p(s)$ of the scaled nearest-neighbor spacing of adiabatic potential curves. $s$ is defined as the nearest-neighbor spacing scaled by the average spacing, at hyper-radius $R=3.8$. Here, $D=100, r_{0}=1$, and $m_{3} / m_{1}=1.3$. States are included from an energy range $-E_{b} \pm E_{\delta}$, where $E_{b}$ is the magnitude of the average lowest dimer-dimer energy at large separation, here and for all the statistical analyses in this paper. For this panel, $E_{\delta}=30$. The dashed curves are the distributions for the Gaussian orthogonal ensemble and the Poisson distribution, respectively. The solid curve plots the kernel density estimator from the level-spacing distribution data, using a Gaussian kernel with bandwidth 0.2. (c) The calculated hyper-radial adiabatic potentials (solid lines) as a function of hyper-radius $R$ at $r_{0}=1, m_{3} / m_{1}=1.3$. $D=10$ (left plot) and $D=100$ (right plot) for the Morse potential. The right plot corresponds to the probability distribution plotted in (b). All length and energy variables are dimensionless, as discussed in Sec. II.

produce converged statistical distributions for potential depths up to $D \sim 75$, for which we retain 160 adiabatic states $\Phi_{\mu}\left(R_{j} ; \Omega\right)$ at each of the $N_{R}=140$ Gauss-Lobatto nodes $R_{j}$. To solve the hyperangular equation (8), B-splines are used as basis functions in both $\theta$ and $\phi$ coordinates. A detailed description is included in Appendix B. Depending on the potential depth, the basis in each angular coordinate is of size 80-160 over the symmetry-restricted domain (see Appendix A).

We first concentrate on the statistical properties of the adiabatic potential curves in the vicinity of the lowest dimer-dimer scattering threshold (Sec. III), and then consider the energy levels of fully bound four-body states (Sec. IV).

\section{NUMERICAL RESULTS FOR ADIABATIC POTENTIALS}

Figure 2(a) displays the two-atom bound states of Morse potential with $D=50$ and $r_{0}=1$ for mass ratios $m_{3} / m_{1}=1$ and $m_{3} / m_{1}=1.3$. For each case, there are 14 bound states. 
Figure 2(c) shows the adiabatic potential curves $U_{v}(R)$ obtained by numerically solving the adiabatic equation at each hyper-radius $R$ and imposing even-parity boundary conditions (see Appendix A for more explanations on the parity symmetry of the system). The left and right plots in Fig. 2(c) correspond to the Morse potential with $D=10$ and $D=$ 100 , respectively. The density of adiabatic potential curves increases as the number of bound states for the two-atom interaction increases. Since our interest is in collisions of ground-state molecules, the vertical axis is scaled by $E_{b}$, which is the absolute value of the average lowest dimerdimer energy at large $R . E_{b}$ is the average of $\left|E_{12}+E_{34}\right|$ and $\left|E_{13}+E_{24}\right|$, i.e., the energies of two possible dimer-dimer configurations. The density of adiabatic potentials increases dramatically as the depth of the model potential increases, predicting a high density of four-body bound states. This will be discussed in detail later.

The statistical distribution of adiabatic potentials $U_{v}(R)$ as a function of $R$ is particularly important for understanding quantum chaos [138-140], and one of the most informative and basic measures of the adiabatic potential statistics is shown in Fig. 2(b). Figure 2(b) shows the probability distribution of nearest-neighbor spacing of the adiabatic potential curves at $R=3.8$, for the $D=100$ case shown in Fig. 2(c). The horizontal axis $s$ is defined as $s=S / \bar{S}$, where $S$ is the nearest-neighbor level spacing, and $\bar{S}$ denotes the average over $S$. At the hyper-radius shown, the level distribution is close to a chaotic distribution, with significant level repulsion readily apparent. 144 curves in an energy range $-E_{b} \pm E_{\delta}$ are included in the statistics, where $E_{b}=$ 181.7 and $E_{\delta}=30$, so that the density of states is approximately uniform over the energy window. The solid curve is the kernel density estimation of the probability distribution given by $p(s)=1 /(\sqrt{2 \pi} n h) \sum_{i=1}^{n} e^{-\left[\left(s-s_{i}\right) / h\right]^{2} / 2}$, where $s_{i}$ are data values and $h=0.2$, an alternative useful to the histogram to estimate $p(s)$. Both methods give consistent $p(s)$ and are relatively insensitive to the bandwidth $h$ and histogram bin size near the values chosen, and none of the conclusions of the paper depend on the details of the choice of method used. The Wigner-Dyson distribution corresponding to a Gaussian orthogonal ensemble and the Poisson distribution, which are predicted to describe chaotic and integrable systems, respectively, are drawn in dashed curves for comparison.

We note that the analysis of the adiabatic potentials $U_{v}(R)$ is a different application of random matrix theory (RMT) than the conventional analysis of the bound state or scattering spectrum [70-72]. One should not necessarily expect, in general, a direct connection between the level statistics of the $U_{v}(R)$ and the bound states. Nevertheless, the level statistics of these curves is worth investigating, for at least two reasons. The first is that it is interesting to search for possible relationships of the $U_{v}(R)$ and the bound state distributions. The second is that the $U_{v}(R)$ are interesting in their own right: for decades, adiabatic hyper-radial potentials have provided both a qualitative and numerical understanding of atomic and molecular scattering. For example, Efimov predicted his now-famous universal three-body states by analyzing these curves [141].

Figure 3 compares the level densities $\rho_{a}$ of the adiabatic potential curves as a function of hyper-radius for different
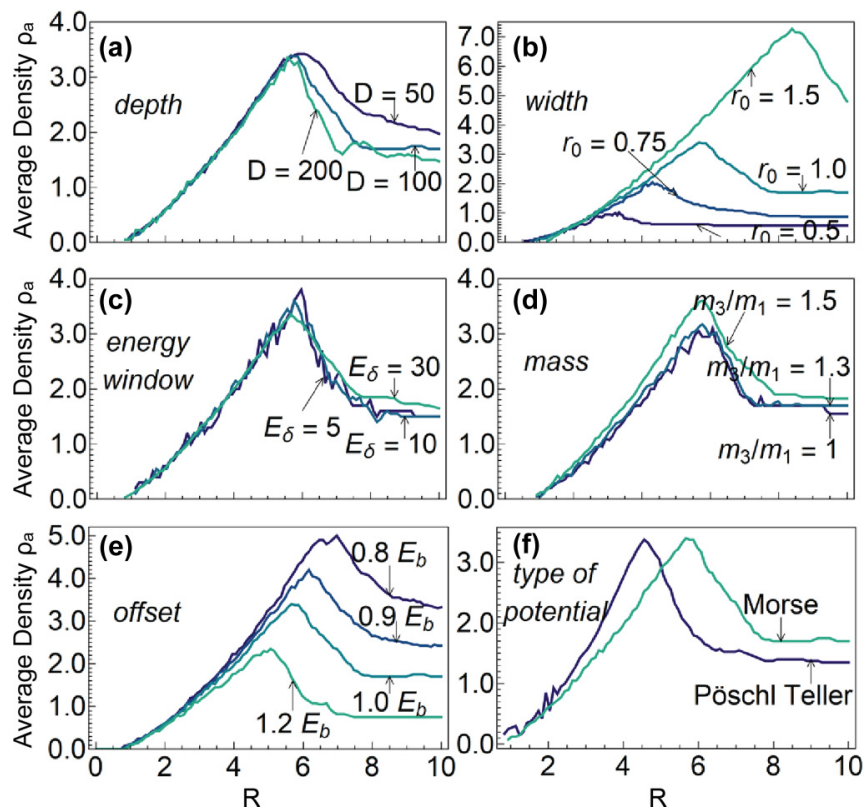

FIG. 3. Illustrations of the level density of adiabatic potential curves $\rho_{a}$ as a function of hyper-radius $R$ for different two-atom interactions. Each plot examines the change in $\rho_{a}$ by varying one parameter of the two-atom interaction. The default nonvarying parameters for (a)-(f) are set to $D=100, r_{0}=1.0, m_{3} / m_{1}=1.3$. The energy range of the included curves is $-E_{b} \pm E_{\delta}$, where $E_{b}=\left(E_{12}+\right.$ $\left.E_{34}+E_{12}+E_{34}\right) / 2=181.7$, and $E_{\delta}=30$. The Morse potential is used in (a)-(e); $r_{0}=2$ for the Pöschl-Teller potential curve is used in (f). (d) $m_{3} / m_{1}=1$. The offset in (e) is defined as the center of the energy range. All length and energy variables are dimensionless, as discussed in Sec. II.

two-atom interactions, and demonstrates a universal trend of $\rho_{a}$ first increasing as $R$ increases, attaining a maximum, and then decreasing to a constant value at long range. The Morse potential was used for Figs. 3(a)-3(e), where it is revealed that $\rho_{a}$ is independent of $D$ and $r_{0}$ for small $R$. This occurs because the kinetic energy dominates in this region, and therefore $\rho_{a}$ is independent of the two-atom interactions. As $R$ increases, $\rho_{a}$ first increases as a result of the decreasing splitting between eigenvalues of the kinetic energy $\left(\propto 1 / R^{2}\right)$, and then decreases and converges to a constant value as the spectrum converges to that of two independent molecules. The peak appears at around $R=6$ for the Morse potential with $r_{0}=1$. Figures 3(a) and 3(b) shows that $\rho_{a}$ decreases (increases) as the depth (width) of the potential increases. For reference, the number of the two-atom bound states for $D=100$ and $D=200$ are 20 and 28, respectively. Figure 3(c) demonstrates that $\rho_{a}$ is independent of the width of energy range $E_{\delta}$ over which the density is calculated for a range of widths from $E_{\delta}=5$ to 30. In Fig. 3(d), no significant shift of $\rho_{a}$ is observed with a change in mass difference. Figure 3(e) shows that $\rho_{a}$ increases as the center of the energy window used to calculate it shifts from low to high $\left(-1.2 E_{b}\right.$ to $\left.-0.8 E_{b}\right)$. This suggests the increase of the complexity of the adiabatic potential curves as the system shifts to higher energies. Figure 3(f) shows the similarity of these features for the Morse and Pöschl-Teller potentials. 

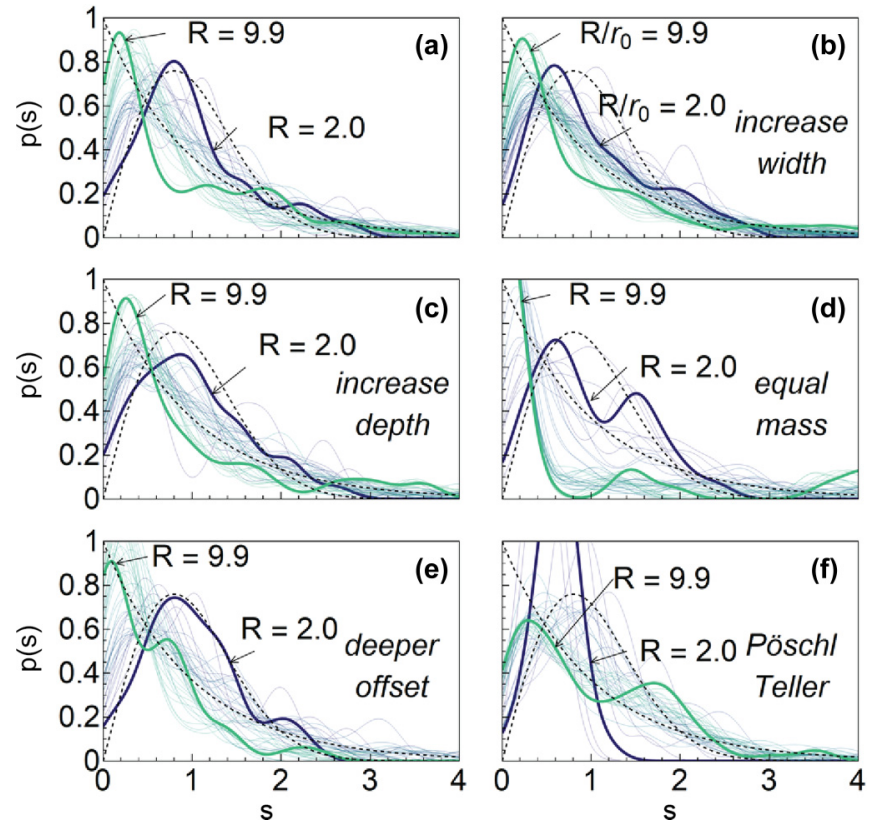

FIG. 4. Probability distribution $p(s)$ of nearest-neighbor spacing of adiabatic potential curves, where $s$ is the scaled spacing, at different hyper-radii $R$. (Kernel density estimator obtained from data with a normal kernel of bandwidth 0.2.) The semitransparent curves are for $R=1.6$ to $R=10$ in steps of $\Delta R=0.2$; the bold curves plot $p(s)$ at $R=9.9$ and $R=2.0$. The dashed curves are the Poisson and GOE distributions in comparison. (a) The result for the Morse potential with $D=100, r_{0}=1$, and $m_{3} / m_{1}=1.3$. The energy range of included curves is taken to be $-E_{b} \pm E_{\delta}$, and $E_{\delta}=30$. (b)-(e) Various cases where one parameter (indicated in each plot) of the two-atom interaction in (a) is modified. (b) The width of the potential characterized by $r_{0}$ is set to $r_{0}=2$. (c) $D=200$. (d) $m_{3} / m_{1}=1$. (e) The center of the included curves is shifted from $E_{b}$ to $E_{b}^{\prime}=1.2 E_{b}=$ 217.6. (f) Using the Pöschl-Teller potential with the same $D, m_{3} / m_{1}$, and $E_{\delta}$ as (a). $r_{0}=2$ is used to keep the numbers of bound states of the two-atom interactions the same for (f) and (a). All length and energy variables are dimensionless, as discussed in Sec. II.

Figure 4 reveals that the nearest-neighbor spacing distribution of adiabatic potentials evolves from the Wigner-Dyson distribution at $R=3.8$ towards a Poisson distribution as $R$ increases. For each panel in Fig. 4, two curves are highlighted by thick lines to represent cases of small and large $R$, and the other lighter curves show other values of $R$ from 1.6 to 10. Each panel shows a different choice of model potential parameters or masses corresponding to the panels in Fig. 3. The chaotic to nonchaotic crossover is clear in Figs. 4(a)-4(c) and 4(e), though with some deviations to be examined later. In Fig. 4(d), a similar trend is visible, with level repulsion and a somewhat Wigner-Dyson-like distribution emerging at small $R$, but at large $R$ the distribution deviates dramatically from Poisson, showing a bunching of levels. Figure 4(f) is the only case where the level repulsion at small $R$ is questionable, showing only a modestly stronger repulsion than for large $R$. We will examine the origins of the features in Figs. 4(d) and 4(f) shortly. (Notice that in these plots, the small dips near $s=0$ that persist to large hyper-radii are artifacts of the Gaussian smooth kernel distribution fitting. A more quantitative
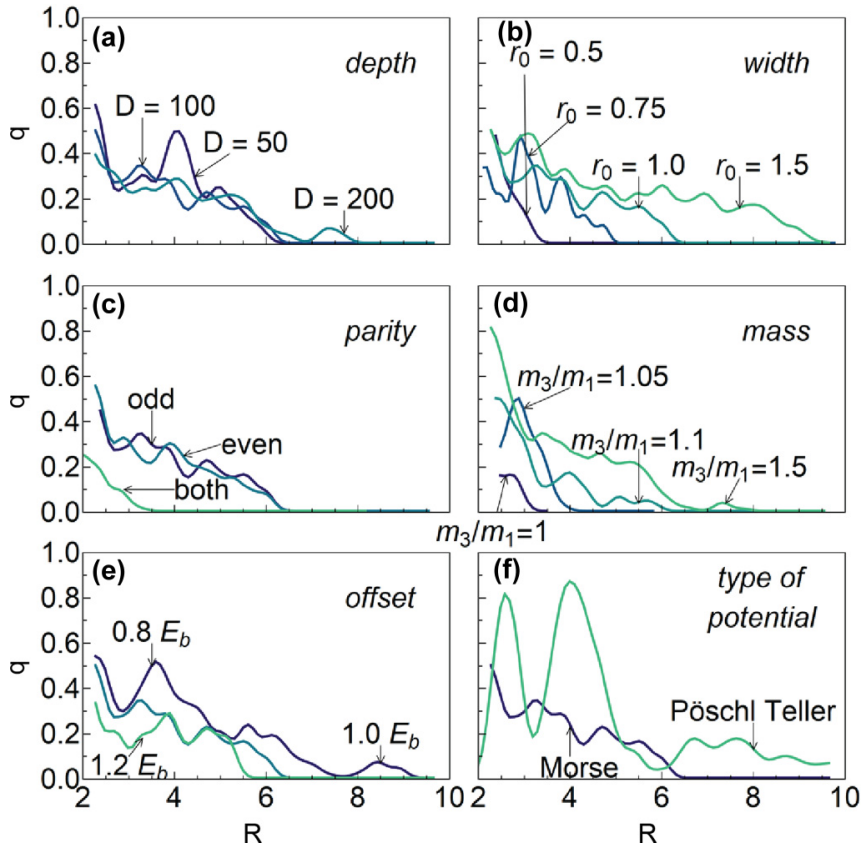

FIG. 5. Brody parameter $q$ as a function of hyper-radius $R$ for different two-atom interactions. Each plot examines the change in $q$ by varying one parameter of the two-atom interaction. The default parameters are the same as those in Fig. 3. Note that the horizontal axis origin starts from $R=2$. Results for different parities are shown in (c). The plots are smoothed via a moving average with weights from a Gaussian distribution. All length and energy variables are dimensionless, as discussed in Sec. II.

measure of the chaoticity of the statistical distribution will be presented later.)

A few other features emerge from close examination of Figs. 4(a)-4(f). In Fig. 4(d), where $m_{3} / m_{1}=1$, a spike near $s=0$ arises for large $R$. This is the result of near degeneracies that occur for large $R$ when $m_{3} / m_{1}=1$. In Fig. 4(f), which shows a Pöschl-Teller potential with $r_{0}=2$, chosen to have the same number of two-atom bound states as Fig. 4(a), there is still a visible level repulsion at small $R$ relative to large $R$, but the shape of the curves is always less well described by both the Wigner-Dyson and Poisson distributions. The difference in the short-range behavior for the Pöschl-Teller case stems from the formation of bands of the adiabatic potential curves at short range. Since at small $R$ the value of the PöschlTeller potential does not vary rapidly, the potential energy surface is nearly constant in the hyperangular coordinates $\theta$ and $\phi$. Hence, the adiabatic potentials separate into bands, where each band is composed of potentials with the same kinetic energy eigenvalue, and the bandwidth - the separation among these potential curves - results from the small differences in their potential energy.

Figure 5 shows a more detailed evaluation of the evolution of chaoticity observed in the level spacing statistics as a function of $R$, by plotting the Brody parameter $q$ [72,142] as a function of the hyper-radius [143]. The Brody distribution is a statistical distribution characterized by $q \in[0,1]$ that can be 
expressed as

$$
P_{\text {Brody }}(s)=\Gamma\left(\frac{2+q}{1+q}\right)^{q+1}(1+q) s^{q} \exp \left(-b s^{q+1}\right) .
$$

It reduces to the standard Poisson distribution at $q=0$ and to Wigner-Dyson at $q=1$. The $q$ plotted in Fig. 5 is determined by a goodness-of-fit hypothesis test to Eq. (16).

Again, all cases shown in Fig. 5 are broadly similar. Take Fig. 5(a) as an example: $q$ smoothly decreases as $R$ increases, at some point falling to zero. The maximum $q$ occurs for small $R$, peaking around $q \sim 0.5$ for $D=100$. Figure 5(a) also shows no significant change in $q$ by changing the twoatom interaction strength over a range from $D=50$ to 200 . Figure 5(b) conveys a similar message that $q$ is unaffected by a change in the width of the two-atom potential from $r_{0}=0.5$ to 1.5 , after $R$ is rescaled by width $r_{0}$. Figure 5(c) shows that $q$ is roughly independent of parity, and that when adiabatic curves of both even and odd parities are included, they become doubly degenerate for $R \gtrsim 4$, leading to a zero effective $q$. At small $R$, the value of $q$ is approximately cut in half when both even and odd parity are included because those sets of spectral lines are uncorrelated with each other, and thus the characteristic level repulsion present in each set separately is not present in the combined set. Figure 5(d) exhibits an increase in $q$ as the difference in the mass of distinguishable fermions increases. Figure 5(e) shows that there is a small increase of $q$ as the system is examined at a higher energy. Figure 5(f) shows that the trends in $q$ for the Pöschl-Teller potential are roughly similar, but $q$ is larger, and with very large oscillations. However, the Pöschl-Teller results are poorly behaved and caution must be exercised in interpreting the results.

\section{FOUR-BODY DENSITY OF BOUND STATES: NUMERICAL RESULTS AND ANALYTIC ESTIMATES}

This section calculates the four-body eigenstates and analyzes their density of states and nearest-neighbor level spacing distributions. For the density of states, we first develop a semiclassical theory, then present the numerical results and compare with the semiclassical theory.

\section{A. Semiclassical analytic estimate of four-body bound state density}

We proceed in the spirit of Ref. [75], with a semiclassical expression for the density of states in all relative degrees of freedom for our four-atom problem. The four-body density of states at energy $E$ classically is

$$
\rho_{4}(E)=\frac{1}{S} \int \frac{d \mathbf{y} d \mathbf{p}}{h^{3}} \delta\left[E-\sum_{i} \frac{p_{i}^{2}}{2 \mu}-V(\mathbf{y})\right] .
$$

$S$ is a symmetry factor that accounts for identical particle symmetry in circumstances where the integral is taken over all phase space. If the integral is restricted to only one region of phase space corresponding to a particular permutation of identical particles, then we set $S \rightarrow 1$. Note that the choice for the four-body reduced mass $\mu=\left(m_{1} m_{2} m_{3} m_{4}\right)^{1 / 4}$ in Eq. (3d) preserves the volume element going from $r_{j}$ to $y_{j}$ coordinates.

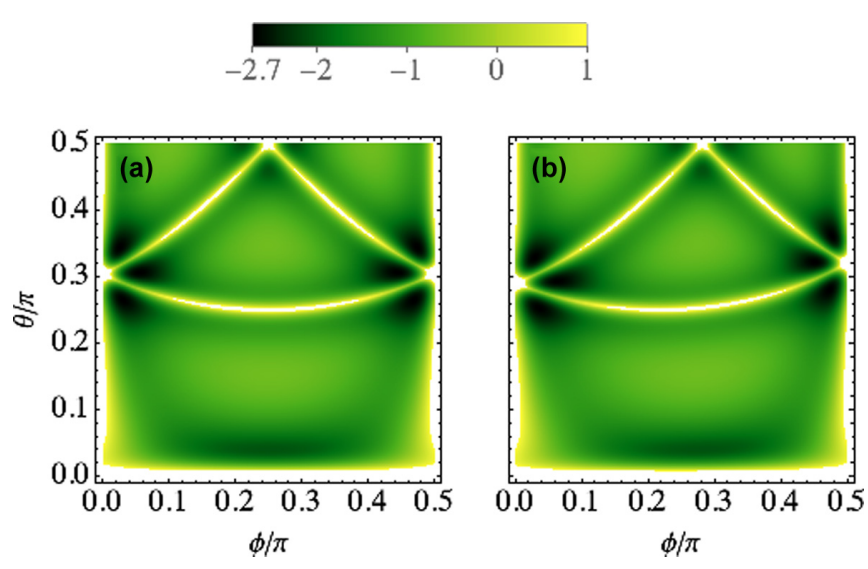

FIG. 6. The hyperspherical potential energy surface in units of the two-body depth $V(R, \theta, \phi) / D$ at $R=8 r_{0}$ for the Morse pairwise potential for (a) equal masses and (b) $m_{3} / m_{1}=1.5$.

It is insightful to evaluate Eq. (17) in (hyper)spherical coordinates (see Sec. II) since all particle permutations leave the hyper-radius invariant. Therefore, $d \mathbf{y} d \mathbf{p}=$ $R^{2} d R d \Omega p^{2} d p d \Omega_{p}$ is the six-dimensional phase-space volume element expressed in spherical polar coordinates. Performing the momentum integral,

$$
\rho_{4}(E)=\frac{(2 \mu)^{3 / 2}}{S 4 \pi^{2} \hbar^{3}} \int R^{2} d R d \Omega \sqrt{E-V(R, \Omega)} .
$$

According to the analysis in Appendix A, coordinate space integrals are reduced to one octant of the total $4 \pi$ solid angle: $\theta \in[0, \pi / 2]$ and $\phi \in[0, \pi / 2]$. This accounts for the $(2 !)^{2}$ ways that identical fermions may be rearranged among themselves, and parity inversion, so with this phase-space restriction, we set $S=1$.

Equation (18) immediately implies two scaling laws: $\rho_{4}(E) \propto \sqrt{D}$ (at fixed $\left.E / D\right)$ and $\rho_{4}(E) \propto r_{0}^{3}$, where $D$ is the depth of the two-body potential and $r_{0}$ is the range of the two-body potential. These can be seen by rewriting in terms of the dimensionless variables $\xi=R / r_{0}, E / D$, and $V / D$ :

$$
\rho_{4}(E)=\mu^{3 / 2} \sqrt{D} r_{0}^{3} \int \mathcal{I}(E, \xi) \xi^{2} d \xi
$$

where

$$
\mathcal{I}(E, \xi)=\frac{1}{\sqrt{2} \pi^{2} \hbar^{3}} \int \sqrt{\frac{E}{D}-\frac{V\left(\xi r_{0}, \Omega\right)}{D}} d \Omega .
$$

The remaining integrals must be evaluated numerically over classically allowed regions in phase space, but note that Eq. (20) is independent of $D$ (at fixed $E / D$ ) since $V\left(\xi r_{0}, \Omega\right) / D$ is independent of $D$. It is also independent of $r_{0}$ since each of the interatomic potentials depends on $r_{0}$ only in the combination $r / r_{0}$. The integral evaluated at each $E / D$ therefore gives the prefactor of the $D$ and $r_{0}$ scaling laws. Figures 6(a) and 6(b) show the potential energy surface $V(R=$ $\left.\xi r_{0}, \Omega\right) / D$ on the hypersphere at $\xi=8$ for the equal-mass and unequal-mass cases, respectively. The most important aspect of the $R$ dependence is the behavior as $R \rightarrow \infty$, where the minimum of $V(R, \Omega)$ approaches a multiple of the two-body potential depth $D$. For Morse pairwise potentials, this minimum turns out to be $V_{\min }(R \rightarrow \infty, \Omega) \rightarrow-2.7 D$. For $E<$ 


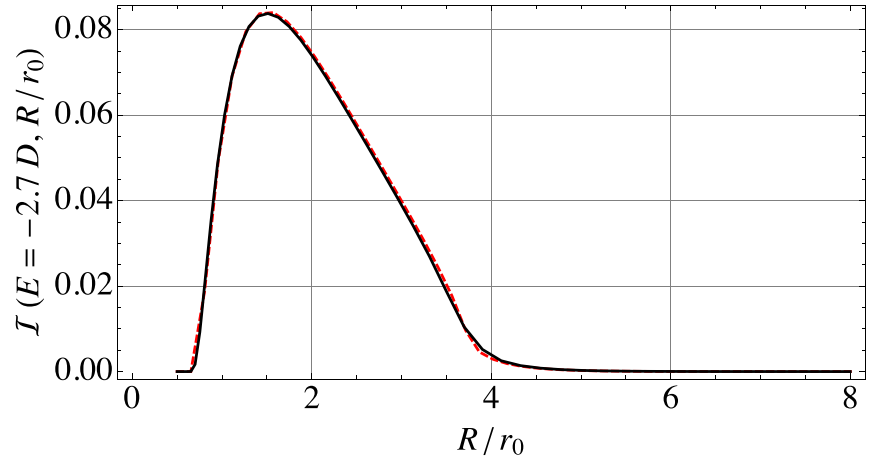

FIG. 7. The hyper-radial function $\mathcal{I}(E=-2.7 D, \xi)$ defined in Eq. (20) for $m_{3}=m_{1}$ (dashed red) and $m_{3}=1.5 m_{1}$ (solid black).

$-2.7 D$, the integral $\mathcal{I}(E, r) \rightarrow 0$ rapidly at sufficiently large $R \gtrsim 4 r_{0}$. For $E \gtrsim-2.7 D$, the integral is dominated by the region $R \lesssim 4 r_{0}$, but has some small contribution from $R \gtrsim 4 r_{0}$ due to the density of the continuum states in open scattering channels.

The four-body density of states at the classical threshold, $E=-2.7 D$, for atoms interacting with the Morse potential, is of particular interest. Figure 7 shows a plot of $\mathcal{I}(E=$ $-2.7 D, \xi)$ for the equal-mass (dashed red) and unequal mass (solid back) cases, revealing that $\mathcal{I}(E, \xi)$ is very nearly independent of the mass ratio. The mass dependence in $\rho_{4}$ is almost entirely captured by the factor of $\mu^{3 / 2}$ that appears in the prefactor of Eq. (19). Note that this near independence of $\mathcal{I}(E, \xi)$ on mass arises because changing the masses only changes the shape of the curves describing particle coalescence in Fig. 6, and not the depth or width of the potential energy valleys. This is expected to persist even if we choose different pairwise interactions between identical particles and distinguishable particles.

\section{B. Numerical calculation of the four-body bound states}

We now compare the semiclassical predictions to our numerical calculations of the four-body bound state density of states. Details regarding the numerical calculation such as the number of Gauss-Lobbato DVR points used and the size of the hyperangular basis set are given in Appendix B.

For Morse pairwise potentials, the integral in Eq. (19) evaluates to $\int_{0}^{\infty} \mathcal{I}(E=-2.7 D, r) r^{2} d r \approx 0.79$. For the case $m_{3}=$ $1.5 m_{1}$, the integral evaluates to 0.78 , due to the slight difference in the curves shown in Fig. 7, and $\mu^{3 / 2} \int \mathcal{I}(r) r^{2} d r=$ 1.06. The resulting scaling laws with $\sqrt{D}$ and $r_{0}^{3}$ are plotted in Fig. 8 for both the equal- and unequal-mass cases. The data points show the density of states calculated from numerical solutions to the Schrödinger equation as $\rho_{4}=\bar{S}^{-1}$, where $\bar{S}$ is the nearest-neighbor level spacing averaged over an energy range $E=-2.7 D \pm 10$. The error bars indicate the standard error in the mean $\delta \rho_{4}=\sigma_{S} /\left(\sqrt{N} \bar{S}^{2}\right)$, where $\sigma_{S}$ is the standard deviation in the set of $N$ level spacings included in the average. Figure 8 clearly demonstrates that all three scaling laws predicted by the semiclassical analysis are confirmed by numerical solutions to the Schrödinger equation. We expect the scaling laws to retain their essential form for the bosonic counterpart to the present two-component Fermi system. The
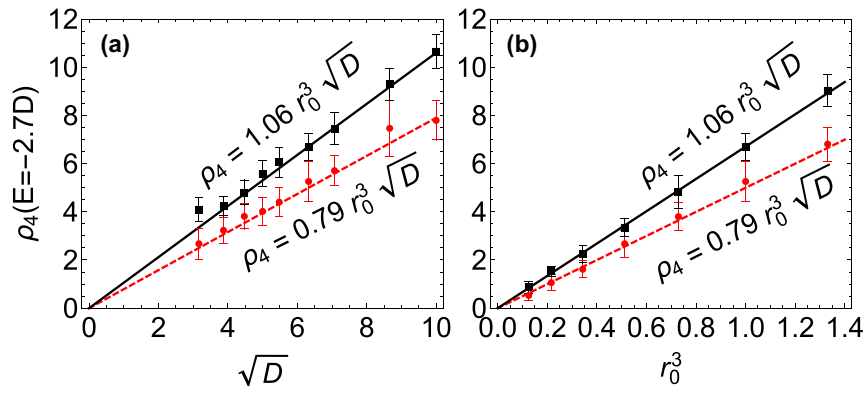

FIG. 8. (a) The agreement between the quantum mechanical four-body bound state density of states and the $\sqrt{D}$ scaling law predicted by the semiclassical theory described in the main text. (b) The scaling with $r_{0}^{3}$. In both panels, the red dashed line and filled circles describe the equal-mass case, while the black solid line and filled squares describe the case $m_{3} / m_{1}=1.5$. The Morse potential was used in all of these calculations, and for (a), we assume $r_{0}=1$, while for (b), we assume $D=40$. All length and energy variables are dimensionless, as discussed in Sec. II.

angular integrals would be carried out over a different domain consistent with bosonic symmetry, but the basic structure of Eq. (19) would remain unchanged.

Finally, we examine the distribution of nearest-neighbor energy level spacings of four-body bound states, and extract a Brody parameter that may serve as a measure of chaos for the four-body collision complex. The fitting to determine this parameter is done using the same procedure as for the adiabatic potential curves.

Figure 9 shows adiabatic potential energy curves, fourbody energy levels, and level spacing statistics for both the equal-mass and unequal-mass problems, both with a pairwise Morse potential of depth $D=75$ and range $r_{0}=1$. Figures 9(a) and 9(b) are for $m_{3}=m_{1}$, while Figs. 9(c) and 9(d) are for $m_{3}=1.5 m_{1}$. Adiabatic potentials are shown as blue curves. Horizontal lines are four-body energy levels. Black (red) lines lie inside (outside) the energy range $E=-2.7 D \pm$ 10 for the statistical analysis for the energy level spacings. The equal-mass problem yields a fairly Poissonian distribution with a Brody parameter $q=0.14$, while the unequal-mass problem yields $q=0.68$.

Since our model uses the same two-body potential for all six interaction terms, the potential energy exhibits an additional symmetry for the equal-mass case that is broken only when the masses are taken to be different. Examination of Fig. 6 reveals that the Hamiltonian for the equal-mass case is invariant under the transformation that takes $\phi \rightarrow \pi / 2-\phi$. This operation amounts to interchanging the magnitudes of the two Jacobi vectors $y_{1}$ and $y_{2}$. The result is that for the equal-mass system, the adiabatic solutions $\Phi_{v}(R, \Omega)$ can be divided into two symmetry classes: those that are even upon $\phi \rightarrow \pi / 2-\phi$ and those that are odd under that operation. The overlap matrix $O_{i v, j \mu}$ from Eq. (13) vanishes between states belonging to different symmetry classes, and one obtains a four-body spectrum that is essentially constructed by overlaying the levels from each class. The resulting distribution shown in Fig. 9(b) looks uncorrelated and accurately fits a Poisson distribution. 

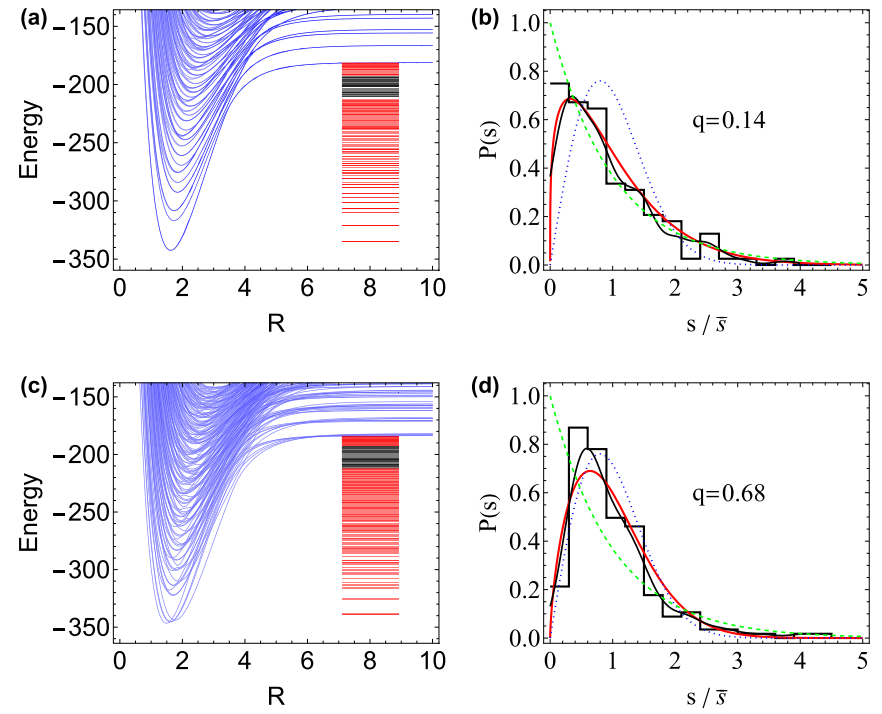

FIG. 9. (a) The adiabatic potentials (blue curves) and the fourbody bound state energy levels (horizontal lines) for the equal-mass case $m_{1}=m_{3}$. Energy levels used to calculate the density of states and the Brody distribution are shown in black, while the remaining energy levels are shown in red. (b) The probability density $P(s)$ of nearest-neighbor energy spacings of four-body bound states, as calculated using the method of slow variable discretization for the equal-mass case. (Black straight lines: histogram; black smooth curve: kernel density estimator.) (c),(d) Corresponding plots for the case $m_{3}=1.5 m_{1}$. The Brody distribution that maximizes the loglikelihood function for the kernel density estimator is shown as the red curve, and panels labeled with the correspondingly fit $q$ value. The blue dotted curves in (b) and (d) show the Wigner-Dyson distribution, while the green dashed curves show the Poisson distribution, included here for comparison. All calculations shown use the Morse pairwise potential with $D=75, r_{0}=1$ and with energy levels in the range $E \pm E_{\delta}=-2.7 D \pm 10$. All length and energy variables are dimensionless, as discussed in Sec. II.

Taking the masses to be different means the potential is no longer symmetric under $\phi \rightarrow \pi / 2-\phi$, as seen in Fig. 6(b). Now, even and odd states under reflection about $\phi=\pi / 4$ couple together. Four-body bound energy levels previously belonging to these separate symmetries are pushed apart by the coupling, resulting in a depletion of small level spacings observed in Fig. 9(d).

\section{CONCLUSIONS}

We have introduced a toy model to understand molecular collisions by considering a 1D system of two identical molecules with identical interactions between each pair of molecules. This allows us to explore a molecular system which has a large density of states in a numerically straightforward fashion. We demonstrate that the hyperspherical adiabatic potentials transition from chaotic (Wigner-Dyson) to nonchaotic (Poisson) with increasing intermolecular separation for certain model potentials, despite the simplicity of the potentials and the reduced dimensionality, and even though the number of two-atom bound states is only $\sim 20$.

We further studied the dependence of the four-atom system's properties on the two-atom interaction by exploring the parameter space of the model potential. We show that the system at short range becomes more Wigner-Dyson-like as the mass ratio of distinguishable particles $m_{3} / m_{1}$ increases. For both the case of $m_{3} / m_{1}=1$ and the case including both inversion parities, the short-range chaos is suppressed by the extra degeneracy in the system. It is found that changing the depth $D$ or width $r_{0}$ of the two-atom interaction within the order of magnitude does not affect the chaoticity of collisions measured by the Brody parameter. The Brody parameter is found to be greater at higher collision energy.

We also calculated and analyzed the four-body density of states $\rho_{4}$ using a full quantum mechanical calculation and a semiclassical approximation. Three scaling laws, governing dependence on $\mu, D$, and $r_{0}$, emerge from the semiclassical analysis, encapsulated in Eq. (19). We found remarkable agreement with the full quantum mechanical calculations.

It will be illuminating to explore realistic collisions in three dimensions. Nevertheless, there are several implications of this study for real molecule scattering. Simple (power-law) relations between the four-body bound state density and twoatom interaction are found and can be explained by a simple theory. We can speculate based on this evidence that the analogous power laws derived in Ref. [65] may accurately predict $\rho_{4}$ for realistic molecule scattering in 3D. If so, this can provide a potent guide for understanding and guiding molecule experiments.

\section{ACKNOWLEDGMENTS}

K.R.A.H. acknowledges support in part from funds from the Welch Foundation, Grant No. C-1872, and the National Science Foundation Grant No. PHY-1848304. K.R.A.H. thanks the Aspen Center for Physics, which is supported by the National Science Foundation Grant No. PHY-1066293, for its hospitality while part of this work was performed.

\section{APPENDIX A: SYMMETRY AND BOUNDARY CONDITIONS}

The exchange symmetry of fermions and spatial inversion symmetry can be used to simplify the problem, by reducing the range of angular coordinates of the Schrödinger equation, and to determine the proper boundary conditions. We can define an operator $\hat{P}_{i j}$ that exchanges the particles $i$ and $j$. Acting $\hat{P}_{12}$ on each of the Jacobi coordinates $y_{i}$ gives

$$
\begin{aligned}
& \hat{P}_{12} y_{1}=-y_{1}, \\
& \hat{P}_{12} y_{2}=y_{2}, \\
& \hat{P}_{12} y_{3}=y_{3} .
\end{aligned}
$$

The hyperspherical coordinates $(R, \theta, \phi)$ transform as

$$
\begin{aligned}
& \hat{P}_{12} R=R, \\
& \hat{P}_{12} \theta=\theta, \\
& \hat{P}_{12} \phi=\pi-\phi .
\end{aligned}
$$

Since particles 1 and 2 are identical fermions, the wave function $\Phi(R, \theta, \phi)$ must be antisymmetric upon exchanging 
particles 1 and 2 . Thus,

$$
\hat{P}_{12} \Phi(R, \theta, \phi)=\Phi(R, \theta, \pi-\phi)=-\Phi(R, \theta, \phi) .
$$

Similarly, for $\hat{P}_{34}$,

$$
\hat{P}_{34} \Phi(R, \theta, \phi)=\Phi(R, \theta,-\phi)=-\Phi(R, \theta, \phi) .
$$

From the above two equations, the wave function defined on $\phi \in[-\pi, \pi)$ can be determined by knowing its value for $\phi \in[0, \pi / 2]$ since, based on Eq. (A4), the value of $\Phi(R, \theta, \phi)$ for $\phi \in[-\pi, 0]$ can be determined by that for $\phi \in[0, \pi]$, and based on Eq. (A3), $\Phi(R, \theta, \phi)$ for $\phi \in[\pi / 2, \pi]$ can be determined by that for $\phi \in[0, \pi / 2]$. From Eqs. (A3) and (A4), the boundary conditions on this reduced region are derived by letting $\phi \rightarrow 0$ or $\phi \rightarrow \pi / 2$, resulting in $\Phi(R, \theta, \phi=0)=0$ and $\Phi(R, \theta, \phi=\pi / 2)=0$.

Spatial inversion symmetry $\hat{\Pi}$ also reduces the coordinate region. This symmetry yields $y_{i} \rightarrow-y_{i}$ for $i=1,2,3$, which corresponds to $(\theta, \phi) \rightarrow(\pi-\theta, \pi+\phi)$. The wave function must be invariant up to a phase under this symmetry, and $\hat{\Pi}^{2}=1$, so

$$
\hat{\Pi} \Phi(R, \theta, \phi)=p \Phi(R, \theta, \phi)=\Phi(R, \pi-\theta, \pi+\phi),
$$

where $p=-1$ for odd parity and $p=1$ for even parity. By arguments similar to those above for the fermionic exchange, Eq. (A5) implies that the range of $\theta$ can be reduced from $[0, \pi]$ to $[0, \pi / 2]$.

Lastly, an inversion of the system's geometry, $y_{3} \rightarrow-y_{3}$, while fixing $y_{1}$ and $y_{2}$ (equivalent to applying $\hat{\Pi} \hat{P}_{12} \hat{P}_{34}$ ), should not alter the boundary conditions for the reduced region, i.e.,

$$
\hat{\Pi} \hat{P}_{12} \hat{P}_{34} \Phi(R, \theta, \phi)=p \Phi(R, \theta, \phi)=\Phi(R, \pi-\theta, \phi) .
$$

Setting $\theta=\pi / 2$, we arrive at another boundary condition: $\left.\Phi(R, \theta, \phi)\right|_{\theta=\pi / 2}=0$ for odd parity and $\left.\frac{\partial}{\partial \theta} \Phi(R, \theta, \phi)\right|_{\theta=\pi / 2}=0$ for even parity.

\section{APPENDIX B: SOLVING THE SCHRÖDINGER EQUATION}

The hyperangular Schrödinger equation,

$$
\begin{aligned}
- & \frac{1}{2 \mu R^{2}}\left(\frac{1}{\sin \theta} \frac{\partial}{\partial \theta} \sin \theta \frac{\partial}{\partial \theta}+\frac{1}{\sin ^{2} \theta} \frac{\partial^{2}}{\partial \phi^{2}}\right) \Phi(R, \theta, \phi) \\
& +V(R, \theta, \phi) \Phi(R, \theta, \phi)=U(R) \Phi(R, \theta, \phi),
\end{aligned}
$$

is solved with B-spline bases [144] for $R, \theta$, and $\phi$, by writing

$$
\Phi(R, \theta, \phi)=\sum_{n} \sum_{m} c_{n, m} u_{n}(\phi) v_{m}(\theta) .
$$

Here, $u_{n}(\phi)$ and $v_{m}(\theta)$ are B-spline functions. First, we insert the B-spline expansion in Eq. (B2) into Eq. (B1) and integrate both sides with $\int d \Omega$ over the unit sphere, yielding

$$
\begin{aligned}
\sum_{n} \sum_{m} c_{n, m} & -\frac{1}{2 \mu R^{2}}\left[s_{\phi}\left(n^{\prime}, n\right) t_{\theta}\left(m^{\prime}, m\right)\right. \\
& \left.+r_{\theta}\left(m^{\prime}, m\right) t_{\phi}\left(n^{\prime}, n\right)\right] \\
& \left.+\int d \Omega u_{n^{\prime}}(\phi) v_{m^{\prime}}(\theta) V(R, \theta, \phi) u_{n}(\phi) v_{m}(\theta)\right\} \\
= & \sum_{n} \sum_{m} c_{n, m} U(R) s_{\phi}\left(n^{\prime}, n\right) s_{\theta}\left(m^{\prime}, m\right),
\end{aligned}
$$

where

$$
\begin{gathered}
s_{\phi}\left(n^{\prime}, n\right)=\int d \phi u_{n^{\prime}}(\phi) u_{n}(\phi) \\
s_{\theta}\left(m^{\prime}, m\right)=\int d \theta \sin \theta v_{m^{\prime}}(\theta) v_{m}(\theta) \\
r_{\theta}\left(m^{\prime}, m\right)=\int d \theta \frac{v_{m^{\prime}}(\theta) v_{m}(\theta)}{\sin \theta} \\
t_{\phi}\left(n^{\prime}, n\right)=\int d \phi u_{n^{\prime}}(\phi) \frac{\partial^{2}}{\partial \phi^{2}} u_{n}(\phi) \\
t_{\theta}\left(m^{\prime}, m\right)=-\int d \theta \sin \theta \frac{\partial v_{m^{\prime}}(\theta)}{\partial \theta} \frac{\partial v_{m}(\theta)}{\partial \theta} .
\end{gathered}
$$

The expression of $t_{\theta}\left(m^{\prime}, m\right)$ is obtained from integration by parts. Values in Eq. (B4) are numerically integrated. B-splines are of the order of 5, and all numerical integrals such as those in Eq. (B4) are evaluated with a Gaussian quadrature with 10 nodes. We choose the numbers of B-splines in the $\theta$ and $\phi$ directions such that the error of the adiabatic potential curves at the largest plotted hyper-radius is $\leqslant 1 \%$ of the average spacing; the number in each direction varies from 100 to 160 depending on the specific parameters of the twoatom interaction. We numerically calculate the eigenvalues and eigenvectors in Eq. (B3) using the Arnoldi algorithm in ARPACK for banded matrices [145].

The four-body bound states are calculated by solving Eq. (12). For a typical calculation with $D=75$ such as the one shown in Fig. 9, we use $N_{R}=120$ radial Gauss-Lobatto grid points (resulting in 118 radial basis functions), and retain $N_{\Phi}=160$ adiabatic eigenstates at each DVR point in Eq. (11), leading to a full (not banded) matrix of size 18880 . Each of the $N_{\Phi}=160$ adiabatic eigenstates is calculated with a product-state B-spline basis as solutions to Eq. (B3). In our convergence tests, we have used values as large as $N_{R}=180$ and $N_{\Phi}=200$, but this results in a negligible change in the density of states and level spacing distribution.
[1] C. P. Koch, M. Lemeshko, and D. Sugny, Quantum control of molecular rotation, Rev. Mod. Phys. 91, 035005 (2019).

[2] B. Gadway and B. Yan, Strongly interacting ultracold polar molecules, J. Phys. B: At., Mol. Opt. Phys. 49, 152002 (2016).
[3] R. Barnett, D. Petrov, M. Lukin, and E. Demler, Quantum Magnetism with Multicomponent Dipolar Molecules in an Optical Lattice, Phys. Rev. Lett. 96, 190401 (2006).

[4] D.-W. Wang, M. D. Lukin, and E. Demler, Quantum Fluids of Self-Assembled Chains of Polar Molecules, Phys. Rev. Lett. 97, 180413 (2006). 
[5] D. Jaksch, V. Venturi, J. I. Cirac, C. J. Williams, and P. Zoller, Creation of a Molecular Condensate by Dynamically Melting a Mott Insulator, Phys. Rev. Lett. 89, 040402 (2002).

[6] M. A. Baranov, K. Osterloh, and M. Lewenstein, Fractional Quantum Hall States in Ultracold Rapidly Rotating Dipolar Fermi Gases, Phys. Rev. Lett. 94, 070404 (2005).

[7] A. Micheli, G. K. Brennen, and P. Zoller, A toolbox for latticespin models with polar molecules, Nat. Phys. 2, 341 (2006).

[8] H. P. Büchler, E. Demler, M. Lukin, A. Micheli, N. Prokof'Ev, G. Pupillo, and P. Zoller, Strongly Correlated 2d Quantum Phases with Cold Polar Molecules: Controlling the Shape of the Interaction Potential, Phys. Rev. Lett. 98, 060404 (2007).

[9] K. Osterloh, N. Barberán, and M. Lewenstein, Strongly Correlated States of Ultracold Rotating Dipolar Fermi Gases, Phys. Rev. Lett. 99, 160403 (2007).

[10] M. A. Baranov, H. Fehrmann, and M. Lewenstein, Wigner Crystallization in Rapidly Rotating 2D Dipolar Fermi Gases, Phys. Rev. Lett. 100, 200402 (2008).

[11] G. M. Bruun and E. Taylor, Quantum Phases of a TwoDimensional Dipolar Fermi Gas, Phys. Rev. Lett. 101, 245301 (2008).

[12] T. Lahaye, C. Menotti, L. Santos, M. Lewenstein, and T. Pfau, The physics of dipolar bosonic quantum gases, Rep. Prog. Phys. 72, 126401 (2009).

[13] J. Quintanilla, S. T. Carr, and J. J. Betouras, Metanematic, smectic, and crystalline phases of dipolar fermions in an optical lattice, Phys. Rev. A 79, 031601(R) (2009).

[14] S.-C. Cheng, Static and dynamical properties of a twodimensional Wigner crystal of rotating dipolar Fermi gases, arXiv:0902.2276.

[15] M. L. Wall and L. D. Carr, Hyperfine molecular Hubbard Hamiltonian, Phys. Rev. A 82, 013611 (2010).

[16] C. Lin, E. Zhao, and W. V. Liu, Liquid crystal phases of ultracold dipolar fermions on a lattice, Phys. Rev. B 81, 045115 (2010).

[17] K. Sun, C. Wu, and S. Das Sarma, Spontaneous inhomogeneous phases in ultracold dipolar Fermi gases, Phys. Rev. B 82, 075105 (2010).

[18] R.-Z. Qiu, S.-P. Kou, Z.-X. Hu, X. Wan, and Su Yi, Quantum Hall effects in fast-rotating Fermi gases with anisotropic dipolar interaction, Phys. Rev. A 83, 063633 (2011).

[19] A. V. Gorshkov, S. R. Manmana, G. Chen, J. Ye, E. Demler, M. D. Lukin, and A. M. Rey, Tunable Superfluidity and Quantum Magnetism with Ultracold Polar Molecules, Phys. Rev. Lett. 107, 115301 (2011).

[20] A. Pikovski, M. Klawunn, A. Recati, and L. Santos, Nonlocal state swapping of polar molecules in bilayers, Phys. Rev. A 84, 061605(R) (2011).

[21] C. Trefzger, C. Menotti, B. Capogrosso-Sansone, and M. Lewenstein, Ultracold dipolar gases in optical lattices, J. Phys. B: At., Mol. Opt. Phys. 44, 193001 (2011).

[22] M. A. Baranov, M. Dalmonte, G. Pupillo, and P. Zoller, Condensed matter theory of dipolar quantum gases, Chem. Rev. 112, 5012 (2012).

[23] N. Y. Yao, C. R. Laumann, A. V. Gorshkov, S. D. Bennett, E. Demler, P. Zoller, and M. D. Lukin, Topological Flat Bands from Dipolar Spin Systems, Phys. Rev. Lett. 109, 266804 (2012).

[24] B. Yan, S. A. Moses, B. Gadway, J. P. Covey, K. R. A. Hazzard, A. M. Rey, D. S. Jin, and J. Ye, Observation of dipolar spin-exchange interactions with lattice-confined polar molecules, Nature (London) 501, 521 (2013).

[25] S. R. Manmana, E. M. Stoudenmire, K. R. A. Hazzard, A. M. Rey, and A. V. Gorshkov, Topological phases in ultracold polar-molecule quantum magnets, Phys. Rev. B 87, 081106(R) (2013).

[26] M. L. Wall, E. Bekaroglu, and L. D. Carr, Molecular Hubbard Hamiltonian: Field regimes and molecular species, Phys. Rev. A 88, 023605 (2013).

[27] M. L. Wall, K. Maeda, and L. D. Carr, Simulating quantum magnets with symmetric top molecules, Annal. Phys. 525, 845 (2013).

[28] M. L. Wall and L. D. Carr, Strongly interacting fermions in an optical lattice, Phys. Rev. A 87, 033601 (2013).

[29] A. V. Gorshkov, K. R. A. Hazzard, and A. M. Rey, Kitaev honeycomb and other exotic spin models with polar molecules, Mol. Phys. 111, 1908 (2013).

[30] M. Lepers, R. Vexiau, M. Aymar, N. Bouloufa-Maafa, and O. Dulieu, Long-range interactions between polar alkali-metal diatoms in external electric fields, Phys. Rev. A 88, 032709 (2013).

[31] K. R. A. Hazzard, B. Gadway, M. Foss-Feig, B. Yan, S. A. Moses, J. P. Covey, N. Y. Yao, M. D. Lukin, J. Ye, D. S. Jin, and A. M. Rey, Many-Body Dynamics of Dipolar Molecules in an Optical Lattice, Phys. Rev. Lett. 113, 195302 (2014).

[32] M. L. Wall, K. Maeda, and L. D. Carr, Realizing unconventional quantum magnetism with symmetric top molecules, New J. Phys. 17, 025001 (2015).

[33] A. K. Fedorov, S. I. Matveenko, V. I. Yudson, and G. V. Shlyapnikov, Novel $p$-wave superfluids of fermionic polar molecules, Sci. Rep. 6, 1 (2016).

[34] B. Sundar, B. Gadway, and K. R. A. Hazzard, Synthetic dimensions in ultracold polar molecules, Sci. Rep. 8, 3422 (2018).

[35] J. Aldegunde Blackmore, L. Caldwell, P. D. Gregory, E. M. Bridge, R. Sawant, J. Aldegunde, J. Mur-Petit, D. Jaksch, J. M. Hutson, B. E. Sauer, M. R. Tarbutt, and S. L. Cornish, Ultracold molecules for quantum simulation: Rotational coherences in CaF and RbCs, Quantum Sci. Technol. 4, 014010 (2018).

[36] L. D. Carr, D. DeMille, R. V. Krems, and J. Ye, Cold and ultracold molecules: Science, technology and applications, New J. Phys. 11, 055049 (2009).

[37] M. Lemeshko, R. V. Krems, J. M. Doyle, and S. Kais, Manipulation of molecules with electromagnetic fields, Mol. Phys. 111, 1648 (2013).

[38] M. L. Wall, K. R. A. Hazzard, and A. M. Rey, From Atomic to Mesoscale: The Role of Quantum Coherence in Systems of Various Complexities (World Scientific, Singapore, 2015).

[39] J. L. Bohn, A. M. Rey, and J. Ye, Cold molecules: Progress in quantum engineering of chemistry and quantum matter, Science 357, 1002 (2017).

[40] D. DeMille, Quantum Computation with Trapped Polar Molecules, Phys. Rev. Lett. 88, 067901 (2002).

[41] A. André, D. DeMille, J. M. Doyle, M. D. Lukin, S. E. Maxwell, P. Rabl, R. J. Schoelkopf, and P. Zoller, A coherent all-electrical interface between polar molecules and mesoscopic superconducting resonators, Nat. Phys. 2, 636 (2006).

[42] S. F. Yelin, K. Kirby, and R. Côté, Schemes for robust quantum computation with polar molecules, Phys. Rev. A 74, 050301(R) (2006). 
[43] F. Herrera, Y. Cao, S. Kais, and K. B. Whaley, Infrared-dressed entanglement of cold open-shell polar molecules for universal matchgate quantum computing, New J. Phys. 16, 075001 (2014).

[44] M. Karra, K. Sharma, B. Friedrich, S. Kais, and D. Herschbach, Prospects for quantum computing with an array of ultracold polar paramagnetic molecules, J. Chem. Phys. 144, 094301 (2016).

[45] K.-K. Ni, T. Rosenband, and D. D. Grimes, Dipolar exchange quantum logic gate with polar molecules, Chem. Sci. 9, 6830 (2018).

[46] M. G. Kozlov and L. N. Labzowsky, Parity violation effects in diatomics, J. Phys. B: At., Mol. Opt. Phys. 28, 1933 (1995).

[47] V. V. Flambaum and M. G. Kozlov, Enhanced Sensitivity to the Time Variation of the Fine-Structure Constant and $m_{p} / m_{e}$ in Diatomic Molecules, Phys. Rev. Lett. 99, 150801 (2007).

[48] J. J. Hudson, D. M. Kara, I. J. Smallman, B. E. Sauer, M. R. Tarbutt, and E. A. Hinds, Improved measurement of the shape of the electron, Nature (London) 473, 493 (2011).

[49] J. Baron, W. C. Campbell, D. DeMille, J. M. Doyle, G. Gabrielse, Y. V. Gurevich, P. W. Hess, N. R. Hutzler, E. Kirilov, I. Kozyryev, B. R. O’Leary, C. D. Panda, M. F. Parsons, E. S. Petrik, B. Spaun, A. C. Vutha, and A. D. West, Order of magnitude smaller limit on the electric dipole moment of the electron, Science 343, 269 (2014).

[50] W. B. Cairncross, D. N. Gresh, M. Grau, K. C. Cossel, T. S. Roussy, Y. Ni, Y. Zhou, J. Ye, and E. A. Cornell, Precision Measurement of the Electron's Electric Dipole Moment Using Trapped Molecular Ions, Phys. Rev. Lett. 119, 153001 (2017).

[51] S. Ospelkaus, K.-K. Ni, D. Wang, M. H. G. de Miranda, B. Neyenhuis, G. Quéméner, P. S. Julienne, J. L. Bohn, D. S. Jin, and J. Ye, Quantum-state controlled chemical reactions of ultracold potassium-rubidium molecules, Science 327, 853 (2010).

[52] K.-K. Ni, S. Ospelkaus, D. Wang, G. Quéméner, B. Neyenhuis, M. H. G. de Miranda, J. L. Bohn, J. Ye, and D. S. Jin, Dipolar collisions of polar molecules in the quantum regime, Nature (London) 464, 1324 (2010).

[53] M. H. G. de Miranda, A. Chotia, B. Neyenhuis, D. Wang, G. Quéméner, S. Ospelkaus, J. L. Bohn, J. Ye, and D. S. Jin, Controlling the quantum stereodynamics of ultracold bimolecular reactions, Nat. Phys. 7, 502 (2011).

[54] N. Balakrishnan, Perspective: Ultracold molecules and the dawn of cold controlled chemistry, J. Chem. Phys. 145, 150901 (2016).

[55] R. V. Krems, Molecules near absolute zero and external field control of atomic and molecular dynamics, Intl. Rev. Phys. Chem. 24, 99 (2005).

[56] R. V. Krems, Cold controlled chemistry, Phys. Chem. Chem. Phys. 10, 4079 (2008).

[57] L. R. Liu, J. D. Hood, Y. Yu, J. T. Zhang, N. R. Hutzler, T. Rosenband, and K.-K. Ni, Building one molecule from a reservoir of two atoms, Science 360, 900 (2018).

[58] M. Guo, X. Ye, J. He, M. L. González-Martínez, R. Vexiau, G. Quéméner, and D. Wang, Dipolar collisions of ultracold ground-state bosonic molecules, Phys. Rev. X 8, 041044 (2018).

[59] E. R. Meyer and J. L. Bohn, Product-state control of bi-alkalimetal chemical reactions, Phys. Rev. A 82, 042707 (2010).
[60] Yu Liu, M.-G. Hu, M. A. Nichols, D. Yang, D. Xie, Hua Guo, and K.-K. Ni, Precision test of statistical dynamics with stateto-state ultracold chemistry, Nature (London) 593, 379 (2021).

[61] G. Quéméner, N. Balakrishnan, and R. V. Krems, Vibrational energy transfer in ultracold molecule-molecule collisions, Phys. Rev. A 77, 030704(R) (2008).

[62] M. Morita, M. B. Kosicki, P. S. Żuchowski, and T. V. Tscherbul, Atom-molecule collisions, spin relaxation, and sympathetic cooling in an ultracold spin-polarized $\mathrm{Rb}\left({ }^{2} s\right)-$ $\operatorname{SrF}\left({ }^{2} \Sigma^{+}\right)$mixture, Phys. Rev. A 98, 042702 (2018).

[63] A. Doçaj, M. L. Wall, R. Mukherjee, and K. R. A. Hazzard, Ultracold Nonreactive Molecules in an Optical Lattice: Connecting Chemistry to Many-Body Physics, Phys. Rev. Lett. 116, 135301 (2016)

[64] M. L. Wall, N. P. Mehta, R. Mukherjee, S. S. Alam, and K. R. A. Hazzard, Microscopic derivation of multichannel Hubbard models for ultracold nonreactive molecules in an optical lattice, Phys. Rev. A 95, 043635 (2017).

[65] M. L. Wall, R. Mukherjee, S. S. Alam, N. P. Mehta, and K. R. A. Hazzard, Lattice-model parameters for ultracold nonreactive molecules: Chaotic scattering and its limitations, Phys. Rev. A 95, 043636 (2017).

[66] E. Ewart, M. L. Wall, and K. R. A. Hazzard, Bosonic molecules in a lattice: Unusual fluid phase from multichannel interactions, Phys. Rev. A 98, 013611 (2018).

[67] M. V. Berry, M. Tabor, and J. M. Ziman, Level clustering in the regular spectrum, Proc. R. Soc. London, Ser. A 356, 375 (1977).

[68] O. Bohigas, M. J. Giannoni, and C. Schmit, Characterization of Chaotic Quantum Spectra and Universality of Level Fluctuation Laws, Phys. Rev. Lett. 52, 1 (1984).

[69] M. L. Mehta, Random Matrices, Pure and Applied Mathematics Series, 3rd ed. (Elsevier, Academic Press, Amsterdam, San Diego, CA, 2004), p. 142.

[70] H. A. Weidenmüller and G. E. Mitchell, Random matrices and chaos in nuclear physics: Nuclear structure, Rev. Mod. Phys. 81, 539 (2009).

[71] G. E. Mitchell, A. Richter, and H. A. Weidenmüller, Random matrices and chaos in nuclear physics: Nuclear reactions, Rev. Mod. Phys. 82, 2845 (2010).

[72] T. A. Brody, J. Flores, J. B. French, P. A. Mello, A. Pandey, and S. S. M. Wong, Random-matrix physics: Spectrum and strength fluctuations, Rev. Mod. Phys. 53, 385 (1981).

[73] M. Mayle, B. P. Ruzic, and J. L. Bohn, Statistical aspects of ultracold resonant scattering, Phys. Rev. A 85, 062712 (2012).

[74] M. Mayle, G. Quéméner, B. P. Ruzic, and J. L. Bohn, Scattering of ultracold molecules in the highly resonant regime, Phys. Rev. A 87, 012709 (2013).

[75] A. Christianen, T. Karman, and G. C. Groenenboom, A quasiclassical method for calculating the density of states of ultracold collision complexes, Phys. Rev. A 100, 032708 (2019).

[76] C. Chin, R. Grimm, P. Julienne, and E. Tiesinga, Feshbach resonances in ultracold gases, Rev. Mod. Phys. 82, 1225 (2010).

[77] A. Frisch, M. Mark, K. Aikawa, F. Ferlaino, J. L. Bohn, C. Makrides, A. Petrov, and S. Kotochigova, Quantum chaos in ultracold collisions of gas-phase erbium atoms, Nature (London) 507, 475 (2014).

[78] T. Maier, I. Ferrier-Barbut, H. Kadau, M. Schmitt, M. Wenzel, C. Wink, T. Pfau, K. Jachymski, and P. S. Julienne, Broad 
universal Feshbach resonances in the chaotic spectrum of dysprosium atoms, Phys. Rev. A 92, 060702 (2015).

[79] T. Maier, H. Kadau, M. Schmitt, M. Wenzel, I. Ferrier-Barbut, T. Pfau, A. Frisch, S. Baier, K. Aikawa, L. Chomaz, M. J. Mark, F. Ferlaino, C. Makrides, E. Tiesinga, A. Petrov, and S. Kotochigova, Emergence of chaotic scattering in ultracold Er and Dy, Phys. Rev. X 5, 041029 (2015).

[80] J. Mur-Petit and R. A. Molina, Spectral statistics of molecular resonances in erbium isotopes: How chaotic are they? Phys. Rev. E 92, 042906 (2015).

[81] K. Jachymski and P. S. Julienne, Analytical model of overlapping Feshbach resonances, Phys. Rev. A 88, 052701 (2013).

[82] K. Jachymski and P. S. Julienne, Chaotic scattering in the presence of a dense set of overlapping Feshbach resonances, Phys. Rev. A 92, 020702(R) (2015).

[83] K. Jachymski, Impact of overlapping resonances on magnetoassociation of cold molecules in tight traps, J. Phys. B: At., Mol. Opt. Phys. 49, 195204 (2016).

[84] C. Makrides, M. Li, E. Tiesinga, and S. Kotochigova, Fractal universality in near-threshold magnetic lanthanide dimers, Sci. Adv. 4, eaap8308 (2018).

[85] L. D. Augustovičová and J. L. Bohn, Manifestation of quantum chaos in Fano-Feshbach resonances, Phys. Rev. A 98, 023419 (2018).

[86] B. C. Yang, J. Pérez-Ríos, and F. Robicheaux, Classical Fractals and Quantum Chaos in Ultracold Dipolar Collisions, Phys. Rev. Lett. 118, 154101 (2017).

[87] D. G. Green, C. L. Vaillant, M. D. Frye, M. Morita, and J. M. Hutson, Quantum chaos in ultracold collisions between $\mathrm{Yb}\left({ }^{1} \mathrm{~S}_{0}\right)$ and $\mathrm{Yb}\left({ }^{3} \mathrm{P}_{2}\right)$, Phys. Rev. A 93, 022703 (2016).

[88] H. Yang, D.-C. Zhang, L. Liu, Y.-X. Liu, J. Nan, B. Zhao, and J.-W. Pan, Observation of magnetically tunable Feshbach resonances in ultracold ${ }^{23} \mathrm{Na}{ }^{40} \mathrm{~K}+{ }^{40} \mathrm{~K}$ collisions, Science $\mathbf{3 6 3}$, 261 (2019).

[89] J. F. E. Croft, C. Makrides, M. Li, A. Petrov, B. K. Kendrick, N. Balakrishnan, and S. Kotochigova, Universality and chaoticity in ultracold $\mathrm{K}+\mathrm{KRb}$ chemical reactions, Nat. Commun. 8, 15897 (2017).

[90] J. F. E. Croft, N. Balakrishnan, and B. K. Kendrick, Longlived complexes and signatures of chaos in ultracold $\mathrm{K}_{2}+\mathrm{Rb}$ collisions, Phys. Rev. A 96, 062707 (2017).

[91] M. A. Nichols, Y.-X. Liu, L. Zhu, Hu M.-G., Y. Liu, and K.$\mathrm{K}$. Ni, Detection of long-lived complexes in ultracold atommolecule collisions, arXiv:2105.14960.

[92] M. D. Frye, M. Morita, C. L. Vaillant, D. G. Green, and J. M. Hutson, Approach to chaos in ultracold atomic and molecular physics: Statistics of near-threshold bound states for $\mathrm{Li}+\mathrm{CaH}$ and Li+CaF, Phys. Rev. A 93, 052713 (2016).

[93] N. P. Mehta, K. R. A. Hazzard, and C. Ticknor, Model for scattering with proliferating resonances: Many coupled square wells, Phys. Rev. A 98, 062703 (2018).

[94] A. Dawid, M. Lewenstein, and M. Tomza, Two interacting ultracold molecules in a one-dimensional harmonic trap, Phys. Rev. A 97, 063618 (2018).

[95] N. P. Mehta and C. D. Morehead, Few-boson processes in the presence of an attractive impurity under one-dimensional confinement, Phys. Rev. A 92, 043616 (2015).

[96] N. P. Mehta, Born-Oppenheimer study of two-component fewparticle systems under one-dimensional confinement, Phys. Rev. A 89, 052706 (2014).
[97] R. C. Forrey, N. Balakrishnan, V. Kharchenko, and A. Dalgarno, Feshbach resonances in ultracold atom-diatom scattering, Phys. Rev. A 58, R2645 (1998).

[98] A. V. Avdeenkov and J. L. Bohn, Ultracold collisions of oxygen molecules, Phys. Rev. A 64, 052703 (2001).

[99] J. L. Bohn, A. V. Avdeenkov, and M. P. Deskevich, Rotational Feshbach Resonances in Ultracold Molecular Collisions, Phys. Rev. Lett. 89, 203202 (2002).

[100] T. V. Tscherbul and R. V. Krems, Controlling Electronic Spin Relaxation of Cold Molecules with Electric Fields, Phys. Rev. Lett. 97, 083201 (2006).

[101] A. Simoni and J.-M. Launay, Ultracold atom-molecule collisions with hyperfine coupling, Laser Phys. 16, 707 (2006).

[102] T. V. Tscherbul, Yu V. Suleimanov, V. Aquilanti, and R. V. Krems, Magnetic field modification of ultracold moleculemolecule collisions, New J. Phys. 11, 055021 (2009).

[103] A. Simoni, J.-M. Launay, and P. Soldán, Feshbach resonances in ultracold atom-molecule collisions, Phys. Rev. A 79, 032701 (2009).

[104] P. S. Żuchowski and J. M. Hutson, Reactions of ultracold alkali-metal dimers, Phys. Rev. A 81, 060703(R) (2010).

[105] J. von Stecher, J. P. D'Incao, and C. H. Greene, Signatures of universal four-body phenomena and their relation to the Efimov effect, Nat. Phys. 5, 417 (2009).

[106] A. Christianen, M. W. Zwierlein, G. C. Groenenboom, and T. Karman, Photoinduced Two-Body Loss of Ultracold Molecules, Phys. Rev. Lett. 123, 123402 (2019).

[107] Yu Liu, M.-G. Hu, M. A. Nichols, D. D. Grimes, T. Karman, H. Guo, and K.-K. Ni, Photo-excitation of long-lived transient intermediates in ultracold reactions, Nat. Phys. 16, 1132 (2020).

[108] P. D. Gregory, J. A. Blackmore, S. L. Bromley, and S. L. Cornish, Loss of Ultracold ${ }^{87} \mathrm{Rb}{ }^{133} \mathrm{Cs}$ Molecules via Optical Excitation of Long-Lived Two-Body Collision Complexes, Phys. Rev. Lett. 124, 163402 (2020).

[109] P. D. Gregory, J. A. Blackmore, M. D. Frye, L. M. Fernley, S. L. Bromley, J. M. Hutson, and S. L. Cornish, Moleculemolecule and atom-molecule collisions with ultracold rbcs molecules, arXiv:2109.08016.

[110] P. D. Gregory, M. D. Frye, J. A. Blackmore, E. M. Bridge, R. Sawant, J. M. Hutson, and S. L. Cornish, Sticky collisions of ultracold RbCs molecules, Nat. Commun. 10, 3104 (2019).

[111] P. Gersema, K. K. Voges, M. Meyer zum Alten Borgloh, L. Koch, T. Hartmann, A. Zenesini, S. Ospelkaus, J. Lin, J. He, and D. Wang, Probing Photoinduced Two-Body Loss of Ultracold Nonreactive Bosonic ${ }^{23} \mathrm{Na}{ }^{87} \mathrm{Rb}$ and ${ }^{23} \mathrm{Na}^{39} \mathrm{~K}$ Molecules, Phys. Rev. Lett. 127, 163401 (2021).

[112] R. Bause, A. Schindewolf, R. Tao, M. Duda, X.-Y. Chen, G. Quéméner, T. Karman, A. Christianen, I. Bloch, and X.-Y. Luo, Collisions of ultracold molecules in bright and dark optical dipole traps, Phys. Rev. Research 3, 033013 (2021).

[113] Z. Z. Yan, J. W. Park, Y. Ni, H. Loh, S. Will, T. Karman, and M. Zwierlein, Resonant Dipolar Collisions of Ultracold Molecules Induced by Microwave Dressing, Phys. Rev. Lett. 125, 063401 (2020).

[114] T. Karman, Z. Z. Yan, and M. Zwierlein, Resonant and firstorder dipolar interactions between ultracold molecules in static and microwave electric fields, arXiv:2106.01610. 
[115] G. Quéméner, J. F. E. Croft, and J. L. Bohn, Electric field dependence of complex-dominated ultracold molecular collisions, arXiv:2109.02602.

[116] J. F. E. Croft, J. L. Bohn, and G. Quéméner, Unified model of ultracold molecular collisions, Phys. Rev. A 102, 033306 (2020).

[117] A. Christianen, G. C. Groenenboom, and T. Karman, Lossy quantum defect theory of ultracold molecular collisions, arXiv:2108.02724.

[118] J. Kłos, Q. Guan, H. Li, M. Li, E. Tiesinga, and S. Kotochigova, Roaming pathways and survival probability in real-time collisional dynamics of cold and controlled bialkali molecules, Sci. Rep. 11, 10598 (2021).

[119] O. I. Tolstikhin, S. Watanabe, and M. Matsuzawa, "Slow" variable discretization: A novel approach for Hamiltonians allowing adiabatic separation of variables, J. Phys. B: At., Mol. Opt. Phys. 29, L389 (1996).

[120] A. Derevianko, E. Luc-Koenig, and F. Masnou-Seeuws, Application of B-splines in determining the eigenspectrum of diatomic molecules: Robust numerical description of halostate and Feshbach molecules, Can. J. Phys. 87, 67 (2009).

[121] H. R. Sadeghpour, J. L. Bohn, M. J. Cavagnero, B. D. Esry, I. I. Fabrikant, J. H. Macek, and A. R. P. Rau, Collisions near threshold in atomic and molecular physics, J. Phys. B: At., Mol. Opt. Phys. 33, R93 (2000).

[122] L. M. Delves, Tertiary and general-order collisions, Nucl. Phys. 9, 391 (1958).

[123] L. M. Delves, Tertiary and general-order collisions (II), Nucl. Phys. 20, 275 (1960).

[124] J. Macek, Properties of autoionizing states of He, J. Phys. B 1, 831 (1968).

[125] D. K. Bondi, J. N. L. Connor, J. Manz, and J. Römelt, Exact quantum and vibrationally adiabatic quantum, semiclassical and quasiclassical study of the collinear reactions $\mathrm{Cl}+\mathrm{MuCl}, \mathrm{Cl}+\mathrm{HCl}, \mathrm{Cl}+\mathrm{DCl}, \mathrm{Mol}$. Phys. 50, 467 (1983).

[126] O. Chuluunbaatar, A. A. Gusev, S. I. Vinitsky, and A. G. Abrashkevich, KANTBP 2.0: New version of a program for computing energy levels, reaction matrix and radial wave functions in the coupled-channel hyperspherical adiabatic approach, Comput. Phys. Commun. 179, 685 (2008).

[127] C. D. Lin, Hyperspherical coordinate approach to atomic and other Coulombic three-body systems, Phys. Rep. 257, 1 (1995).

[128] H. Suno, B. D. Esry, C. H. Greene, and J. P. Burke, Three-body recombination of cold helium atoms, Phys. Rev. A 65, 042725 (2002).

[129] E. Nielsen, D.V. Fedorov, A. S. Jensen, and E. Garrido, The three-body problem with short-range interactions, Phys. Rep. 347, 373 (2001).

[130] B. D. Esry, C. D. Lin, and C. H. Greene, Adiabatic hyperspherical study of the helium trimer, Phys. Rev. A 54, 394 (1996).
[131] N. P. Mehta, S. T. Rittenhouse, J. P. D’Incao, J. von Stecher, and C. H. Greene, General Theoretical Description of $n$-Body Recombination, Phys. Rev. Lett. 103, 153201 (2009).

[132] S. T. Rittenhouse, J. von Stecher, J. P. D'Incao, N. P. Mehta, and C. H. Greene, The hyperspherical four-fermion problem, J. Phys. B 44, 172001 (2011).

[133] J. V. Lill, G. A. Parker, and J. C. Light, Discrete variable representations and sudden models in quantum scattering theory, Chem. Phys. Lett. 89, 483 (1982).

[134] F. W. Olver, D. W. Lozier, R. F. Boisvert, and C. W. Clark, NIST Handbook of Mathematical Functions (Cambridge University Press, New York, 2010).

[135] T. N. Rescigno and C. W. McCurdy, Numerical grid methods for quantum-mechanical scattering problems, Phys. Rev. A 62, 032706 (2000).

[136] H. Suno, Hyperspherical slow variable discretization method for weakly bound triatomic molecules, J. Chem. Phys. 134, 064318 (2011).

[137] D. E. Manolopoulos and R. E. Wyatt, Quantum scattering via the log derivative version of the Kohn variational principle, Chem. Phys. Lett. 152, 23 (1988).

[138] T.-N. Chang, Many-Body Theory of Atomic Structure and Photoionization (World Scientific, Singapore, 1993).

[139] G. Capecchi, D. De Fazio, G. Grossi, L. Peroncelli, and N. Rahman, Dynamics on reactive potential energy surfaces: Hyperspherical view and signatures of "quantum chaos", Mol. Phys. 99, 443 (2001).

[140] K. M. Daily, A. Kievsky, and C. H. Greene, Adiabatic hyperspherical analysis of realistic nuclear potentials, Few-Body Syst. 56, 753 (2015).

[141] V. Efimov, Energy levels arising from resonant two-body forces in a three-body system, Phys. Lett. B 33, 563 (1970).

[142] T. A. Brody, A statistical measure for the repulsion of energy levels, Lett. Nuovo Cimento 7, 482 (1973).

[143] In Fig. 5, because the potential curves are selected from a specific energy range, $q$ has rapid oscillations as $R$ changes, as levels move in and out of the selected energy window. A moving average is applied to smooth the curves to determine their general feature. The average $\left(R_{i}, q_{i}\right)$ is given by $R_{i}=\sum_{n=i}^{n=i+9} f_{n} R_{n}$ and $q_{i}=\sum_{n=i}^{n=i+9} f_{n} q_{n}$, where $R_{n}$ and $q_{n}$ are the hyper-radii and Brody parameter evaluated at grid points indexed by $n$, with adjacent $R_{n}$ separated by $\Delta R=0.1 . f_{n}$ are weights given by a normalized Gaussian with width 1.58.

[144] C.-N. Liu, A.-T. Le, T. Morishita, B. D. Esry, and C. D. Lin, Hyperspherical close-coupling calculations for charge-transfer cross sections in $\mathrm{He}^{2+}+\mathrm{H}(1 s)$ collisions at low energies, Phys. Rev. A 67, 052705 (2003).

[145] R. B. Lehoucq, D. C. Sorensen, and C. Yang, ARPACK Users' Guide: Solution of Large-Scale Eigenvalue Problems with Implicitly Restarted Arnoldi Methods (Society for Industrial and Applied Mathematics, Philadelphia, 1998). 